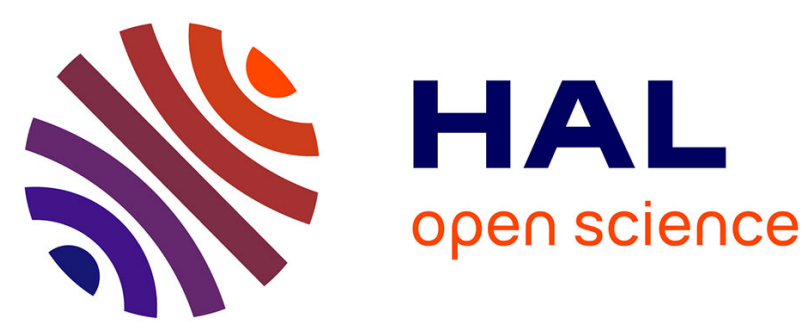

\title{
Chiral Synthetic Equivalents of 2-Cyanoethyl Tetraisopropylphosphorodiamidite: Application to the Synthesis and Resolution of Chiral Phosphoric Acids
}

Kevin Isaac, Jérémie Stemper, Pascal Retailleau, Jean-François Betzer, Angela Marinetti

\section{To cite this version:}

Kevin Isaac, Jérémie Stemper, Pascal Retailleau, Jean-François Betzer, Angela Marinetti. Chiral Synthetic Equivalents of 2-Cyanoethyl Tetraisopropylphosphorodiamidite: Application to the Synthesis and Resolution of Chiral Phosphoric Acids. European Journal of Organic Chemistry, 2014, 2014 (19), pp.4099-4106. 10.1002/ejoc.201402203 . hal-02325095

\section{HAL Id: hal-02325095 \\ https://hal.science/hal-02325095}

Submitted on 23 Oct 2019

HAL is a multi-disciplinary open access archive for the deposit and dissemination of scientific research documents, whether they are published or not. The documents may come from teaching and research institutions in France or abroad, or from public or private research centers.
L'archive ouverte pluridisciplinaire HAL, est destinée au dépôt et à la diffusion de documents scientifiques de niveau recherche, publiés ou non, émanant des établissements d'enseignement et de recherche français ou étrangers, des laboratoires publics ou privés. 


\title{
Chiral Synthetic Equivalents of 2-Cyanoethyl Tetraisopropylphosphorodiamidite: Application to the Synthesis and Resolution of Chiral Phosphoric Acids
}

\author{
Kévin Isaac, ${ }^{[a]}$ Jérémie Stemper, ${ }^{[a]}$ Pascal Retailleau, ${ }^{[a]}$ Jean-François Betzer ${ }^{[a] *}$ \\ and Angela Marinetti ${ }^{[a]^{*}}$
}

Keywords: chiral phosphorodiamidites / resolution / phosphoric acids / phosphates.

Four synthetic equivalents of $\left(i \mathrm{Pr}_{2} \mathrm{~N}\right)_{2} \mathrm{P}-\mathrm{OCH}_{2} \mathrm{CH}_{2} \mathrm{CN}, \mathbf{1}$, have been prepared from easily available enantiomerically pure $\beta$-hydroxynitriles as well as from chiral $\beta$-hydroxyesters. To demonstrate their possible use as phosphinating/resolving agents, these phosphorodiamidites have been used in the synthesis of known phosphoric acids. The method allows enantiomerically pure acids to be obtained via separation of the diastereomeric phosphates. The method should favourably compete with other resolution procedures, e.g. fractional crystallisation or chiral HPLC, for the small scale synthesis of new phosphoric acids for screening purposes. As synthetic equivalents of $\mathbf{1}$, these auxiliaries might find applications also in nucleoside chemistry for the synthesis of biologically relevant compounds. [a] Institut de Chimie des Substances Naturelles, CNRS UPR $2301-$ Centre de Recherche de Gif - 1, av. de la Terrasse 91198 Gif-sur-Yvette, France

E-mail: angela.marinetti@cnrs.fr jean-francois.betzer@cnrs.fr

Homepage: http://www.icsn.cnrs-gif.fr/

Supporting information for this article is available on the WWW under http://dx.doi.org/10.1002/ejoc.201402203

\section{Introduction}

The commercially available tetraisopropyl 2-cyanoethylphosphorodiamidite $\mathbf{1}$ is a common reagent for the generation of cyanoethyl phosphates $(\mathrm{RO})_{2} \mathrm{P}(\mathrm{O})\left(\mathrm{OCH}_{2} \mathrm{CH}_{2} \mathrm{CN}\right)$ from alcohols or diols, mainly used bioorganic chemistry e.g. in carbohydrates, nucleotides and phospholipids chemistry. From these phosphates, the cyanoethyl group can be easily removed via base-induced elimination of acrylonitrile, to generate the corresponding phosphoric acids, phosphodiesters and others. ${ }^{[1][2]}$ Chiral variants of phosphorodiamidite $\mathbf{1}$ might be highly useful auxiliaries for asymmetric syntheses via either diastereoselective processes or diastereomers separation. Chiral variants of this reagent could be prepared, in principle, from a variety of chiral alcohols bearing electron-withdrawing groups in their $\beta$-positions (Figure 1), but compounds of this class have not been described in the literature.

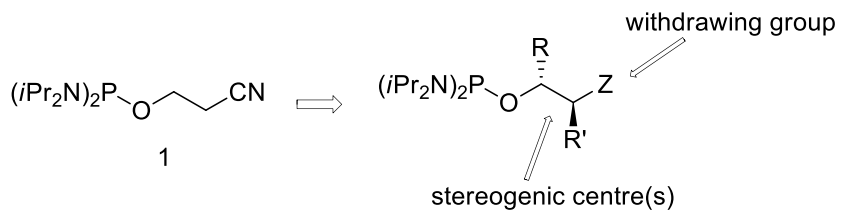

We report here on the synthesis of new, structurally diverse chiral $N, N, N, N$-tetraisopropyl phosphorodiamidites that are easily available from chiral pool substrates. We demonstrate their use as synthetic equivalents of cyanoethyl phosphorodiamidite $\mathbf{1}$, both as phosphorylating agents and precursors for $\mathrm{P}(\mathrm{O})-\mathrm{OH}$ functions.

The use of these phosphorodiamidites has been typified here by a rather non-conventional application, i.e. the synthesis and resolution of chiral phosphoric acids, following to our recent work in this field. ${ }^{[3]}$ However, synthetic uses of these chiral reagents in many different applications can be easily anticipated.

\section{Results and Discussion}

Chiral phosphoric acids and the related phosphate salts have turned recently into highly useful auxiliaries and catalysts for a variety of enantioselective processes. ${ }^{[4]}{ }^{[5]}$ Chiral phosphoric acids are mainly accessed from enantiomerically pure diols, e.g. Spinol, ${ }^{[6]}$ BINOLs, ${ }^{[7]}$ VANOL and VAPOL. ${ }^{[8]}$ Alternatively, enantiomeric resolution of the phosphoric acid themselves may rely on crystallization of diastereomeric salts, e.g. brucine or cinchonidine derivatives, ${ }^{[9]}$ or preparative HPLC separation of enantiomers. Crystallization processes are perfectly suited for large scale syntheses, while chiral HPLC, although expensive, is well adapted for small scale production of chiral samples for screening purposes. We propose here an alternative approach to phosphoric acids taking advantage of chiral auxiliaries, covalently bonded to the phosphorus atom via P-OR functions, and chromatographic separation of epimeric phosphites, or the corresponding phosphates (Figure 2). 


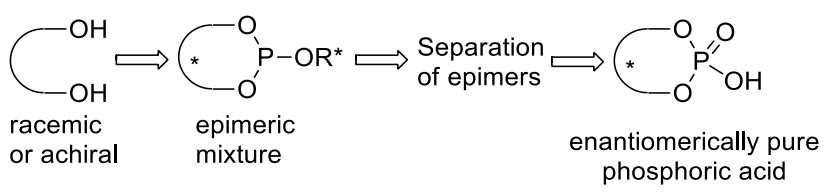

Figure 2. Proposed synthesis and resolution procedure

For such purpose, the chiral auxiliary must be easily removable so as to convert the intermediate phosphite epimers into the desired acid in a few steps. Surprisingly, this very intuitive strategy has not been applied so far to the synthesis and resolution of phosphoric acid precursors.

Recently, during our studies on the synthesis of chiral phosphoric acids based on paracyclophane scaffolds shown in Scheme $1 b,{ }^{[3 a]}$ we have faced a crucial issue: the diol precursors being achiral, the common strategy of resolving the diol precursors couldn't be applied in this series. Although chiral HPLC allowed resolution of the final chiral acids, this technique prevented however an easy and economically viable scale-up. This led us to envision the design of chiral phosphinating agents as synthesis/resolution tools, according to the strategy displayed in Figure 2 above. Thus, at the outset of this study, we have considered the (S)-3-hydroxy-3phenylpropanenitrile-derived phosphorodiamidite $(S)-\mathbf{4}$ as a chiral synthetic equivalent of $\mathbf{1}$ (Scheme 1a), i.e. as a precursor for the phosphoric acid function.

Nitrile $(S)-\mathbf{3}$ is easily available from the enantiomerically enriched (R)-1-phenyl-2-chloroethanol 2: conversion of chloride $\mathbf{2}$ into nitrile 3 by means of $\mathrm{KCN}$ occurs in $74 \%$ yield at a $7 \mathrm{~g}$ scale. Then, addition of bis(diisopropylamino)chlorophosphine to $(S)$-3 in the presence of triethylamine affords the desired cyanoethyl-phosphorodiamidite $(S)$-4. Purification of this compound has been performed by quick filtration on a silica gel column with heptane/EtOAc (7:3) / $\mathrm{Et}_{3} \mathrm{~N}$ $(1 \%)$ as the eluent. $(S)-\mathbf{4}$ has been obtained in $78 \%$ yield in multigram scale, as a stable, easy to handle crystalline solid.

This chiral auxiliary has been used then in the synthesis and resolution of paracyclophane-based phosphoric acids as recalled in Scheme $1 b .^{[3 b]}$ (a)

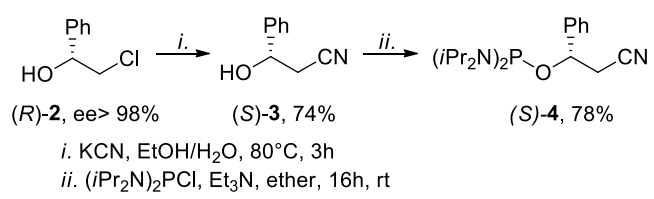

(b)

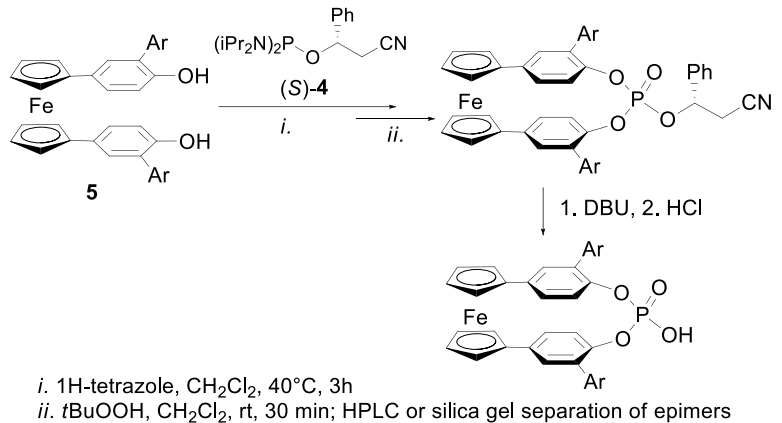

Scheme 1. (a) Synthesis of the chiral phosphorodiamidite $(S)-\mathbf{4}$ and (b) use for the synthesis of paracyclophane-type phosphates. ${ }^{[3 \mathrm{~b}]}$

We illustrate here the potential usefulness of $(S)-\mathbf{4}$, beyond the initially envisioned application, by typifying its use in the synthesis of some of the currently most popular chiral acids. As shown in Scheme 2, phosphorodiamidite $(S)-\mathbf{4}$ reacts with BINOL, VANOL and VAPOL to afford mixtures of the corresponding epimeric phosphites. Phosphites being rather air-sensitive, separation of the epimers is better performed on the corresponding phosphates 6 which are obtained after oxidation of phosphorus with $t$ butylhydroperoxyde. In all cases, pairs of epimers are easily separated by preparative HPLC at a $0.1 \mathrm{~g}$ scale in the conditions given in Scheme 2. The single epimers of phosphates $\mathbf{6}$ have been fully characterized. The relative configurations of these phosphates have been assigned afterward, from the sign of the optical rotation of the corresponding acids $7 \mathbf{a}-\mathbf{c}^{[8 \mathrm{a}, 10]}$

Finally, the cyanoethyl group has been removed by treatment with DBU, at room temperature in dichloromethane. The pure acids 7 have been isolated after filtration of the DBU salts on a short silica gel column (removal of excess DBU and cinnamonitrile) and washing of the salt with a concentrated $\mathrm{HCl}$ solution.

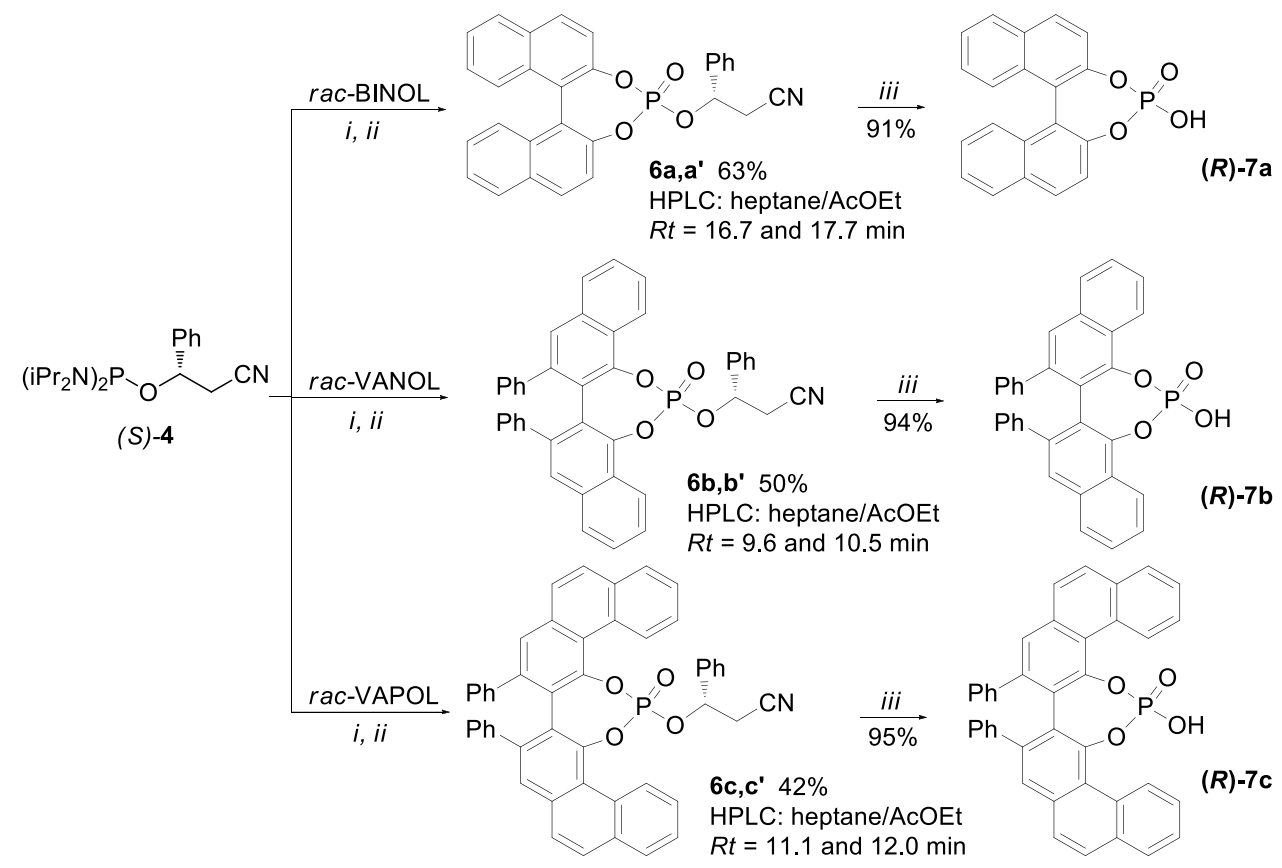

i. $1 \mathrm{H}$-tetrazole, 1,2 -dichloroethane, $3 \mathrm{~h}, 80^{\circ} \mathrm{C}$

ii. TBHP, $\mathrm{CH}_{2} \mathrm{Cl}_{2}, 0-25^{\circ} \mathrm{C}, 1 \mathrm{~h}$

iii. (a) $\mathrm{DBU}, \mathrm{CH}_{2} \mathrm{Cl}_{2}$, r.t., 20 min; (b) $\mathrm{HCl}_{\mathrm{aq}}$ 
Beyond the atropisomeric biaryl-derivatives in Scheme 2, the method is illustrated also by the efficient separation of the spiranic phosphates 6d,d', which are precursors for the spinol derived acid $\mathbf{7 d}^{[6 b]}$ (Scheme 3). In this case, separation of the epimers does not require HPLC techniques, it can be carried out by column chromatography on silica gel (toluene/THF 9:1, $R_{\mathrm{f}}=0.31$ and 0.25 ). The $(R, S)$-phosphate $\mathbf{6 d}$ has been characterized by an X-ray crystal diffraction study. An ORTEP drawing for this compound is displayed in Scheme 3, showing that the spinol scaffold of $\mathbf{6 d}$ has an $(R)$-configuration.

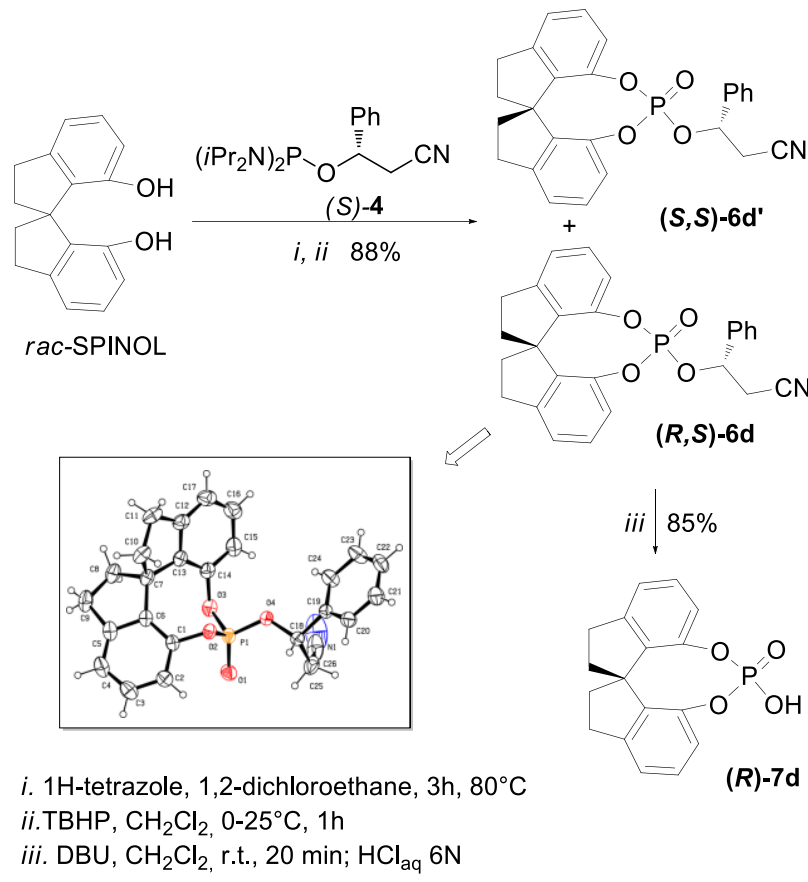

Scheme 3. Synthesis of the SPINOL-derived phosphoric acids 7d via the chiral auxiliary (S)-4

Phosphates 6d and 6d' have been converted into the corresponding acids by reaction with DBU, under the above conditions.

The few experiments above validate our design of $(S)-\mathbf{4}$ as a chiral auxiliary in the synthesis of phosphoric acids, as far as $(S)-\mathbf{4}$ is readily available and allows easy separation of the diastereomeric phosphates in these four cases. Obviously, phosphates made by combining (S)-4 with other chiral scaffolds might challenge however chromatographic separations. Therefore, to ensure a general applicability of the proposed method (Figure 2), it would be preferable to have at our disposal a larger set of chiral auxiliaries.

With this aim in mind, we have considered some new phosphorodiamidites as potential resolution agents (Scheme 4). Thus, we have prepared at first the $(R)$-ethyl 4-cyano-3hydroxybutanoate derived phosphorodiamidite $(R)-\mathbf{8}$. This compound was anticipated to afford suitable phosphoric acid precursors, given its structural analogy to $(S)-\mathbf{4}$ related to the presence of the $\beta$-cyano-function. We have also envisioned that the range of phosphinating/resolving agents might be extended to phosphorodiamidites bearing withdrawing functions other than cyanides in their $\beta$-positions. To check this point, we have prepared the new phosphorodiamidites $\mathbf{9}$ and $\mathbf{1 0}$ which display esters as the withdrawing functions (Scheme 4). These compounds have the main advantage of being easily obtained from commercially available, chiral pool substrates, i.e. malic acid and threonine esters respectively.

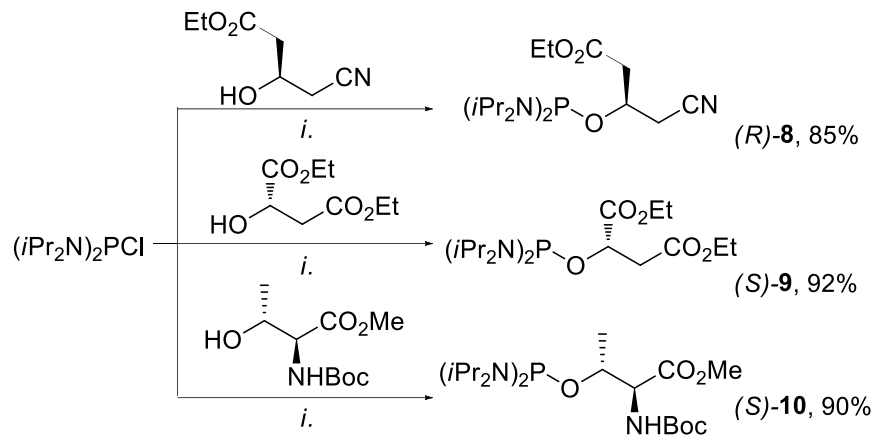

i. $\mathrm{Et}_{3} \mathrm{~N}$, ether, $0^{\circ} \mathrm{C}$ to $\mathrm{rt}$

Scheme 4. Synthesis of the chiral phosphorodiamidites 8-10.

Phosphorodiamidites 8-10 have been obtained in good yields from the corresponding chiral alcohols ${ }^{[1]]}$ by reaction with $\left(i \mathrm{Pr}_{2} \mathrm{~N}\right)_{2} \mathrm{PCl}$ and have been used most often without further purification. If needed, they can be purified by filtration on a short alumina column.

The three phosphorodiamidites 8-10 have been tested initially as chiral resolving agents in the synthesis of planar chiral phosphoric acids with paracyclophane structures (Scheme 5). During these studies, we succeeded in the synthesis of the desired phosphates 1113 [11 a,a': $\delta^{31} \mathrm{P}=-14.9$ and -15.2 ppm (1:1 ratio); 12a,a': $\delta^{31} \mathrm{P}=$ 14.0 and -14.5 ppm (6:4 ratio); 13a,a': $\delta^{31} \mathrm{P}=-13.2$ and $-14.5 \mathrm{ppm}$ ( $2: 8$ ratio)] but unfortunately we couldn't find suitable conditions for the separation of these epimeric mixtures. ${ }^{[12]}$

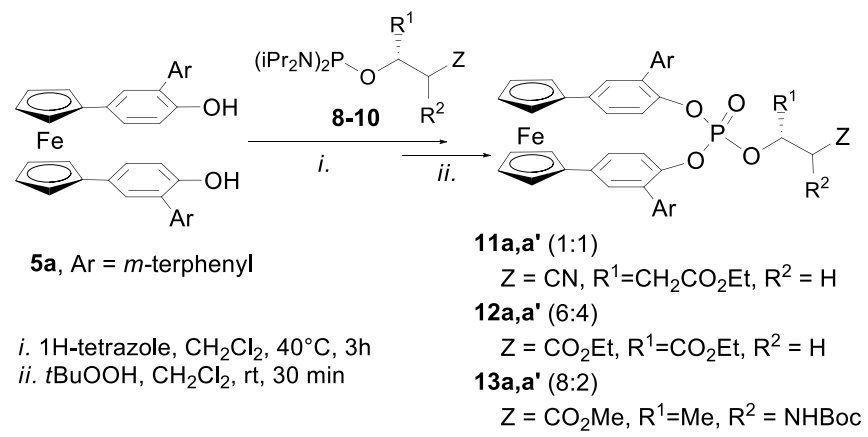

Scheme 5. (a) Synthesis of epimeric mixtures of phosphates 10-12.

Nevertheless, an interesting point has been noticed during these studies: the cyclization reaction between the ferrocenic diol 5a and the threonine-derived phosphorodiamidite $(S)$-10 generates the cyclic phosphates 13a,a' as a 8:2 epimeric mixture, i.e. with a significant level of chiral induction at the ring closing step. Although the stereoselectivity level of these cyclizations must still be improved, this result opens the way to asymmetric syntheses of this class of chiral paracyclophanic derivatives. 
Next, to check if phosphorodiamidites 8-10 can behave as chiral equivalents of $\mathbf{4}$ in the synthesis of phosphoric acids, we have considered VANOL as the model diol. All three phosphorodiamidites react with VANOL in the presence of $1 \mathrm{H}$ tetrazole in mild conditions to give the desired phosphites that are converted in situ into phosphates 14-16.

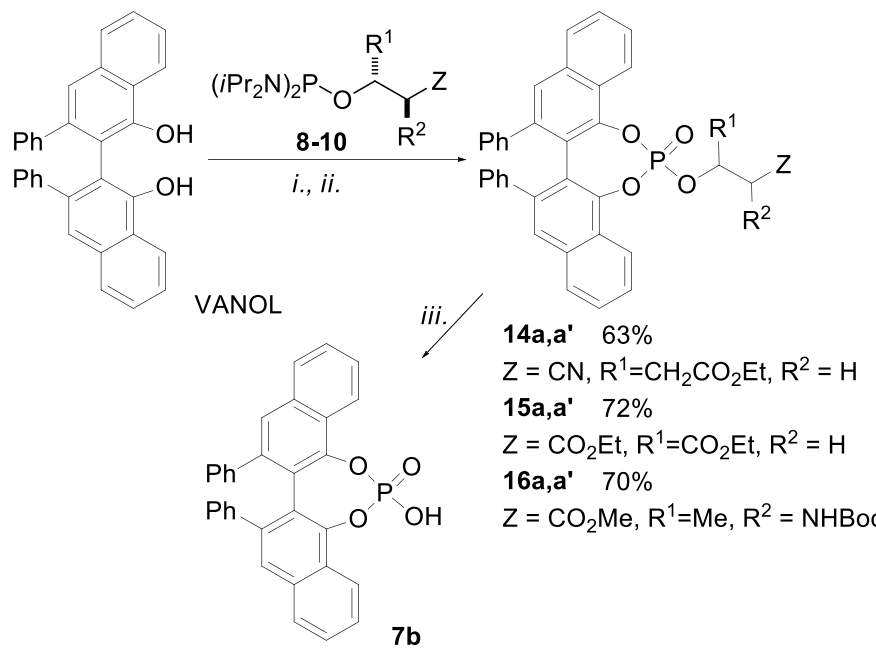

i. $1 \mathrm{H}$-tetrazole, $\mathrm{CH}_{2} \mathrm{Cl}_{2}, 16 \mathrm{~h}, 40^{\circ} \mathrm{C}$

or $1 \mathrm{H}$-tetrazole, 1,2 -dichloroethane, $4 \mathrm{~h}, 80^{\circ} \mathrm{C}$ (for $14 \mathrm{a}, \mathrm{a}^{\prime}$ )

ii. TBHP, $\mathrm{CH}_{2} \mathrm{Cl}_{2}, 0^{\circ} \mathrm{C}$ to r.t.

iii. (a) $\mathrm{DBU}, \mathrm{rt}, 20 \mathrm{~min}$. (b) $\mathrm{HCl}_{\mathrm{aq}}$

Scheme 6. Synthesis of the VANOL-derived phosphoric acid $7 \mathbf{b}$ via the chiral auxiliaries 8-10.

The epimers of phosphates 14-16 have been separated by HPLC under the conditions specified in the experimental section. Finally, all three chiral chains, including the amino-acid derived chain of $\mathbf{1 6}$, could be removed from the intermediate phosphates in the presence of DBU, leading to the VANOL derived phosphoric acid $\mathbf{7 b}$. These experiments demonstrate that the ester functions of $\mathbf{9}$ and $\mathbf{1 0}$ play the same role as the cyano-function in $\mathbf{1}$ by favoring deprotonation of the $\beta$-hydrogen atom and elimination of the corresponding olefins. Thus, phosphorodiamidites 8-10 can be envisioned for further uses as suitable, new chiral synthetic equivalents of the cyanoethyl phosphorodiamidite $\mathbf{1}$.

\section{Conclusion}

The successful experiments in Scheme 4 and Scheme 6 expand the scope of our new strategy of using chiral auxiliaries for the synthesis/resolution of phosphoric acids. They demonstrate that a variety of chiral-pool derived phosphorodiamidites, easily available in enantiomerically pure form, can be used for this purpose and provide therefore a suitable toolbox. As far as it offers a suitable resolution procedure, this new synthetic method may facilitate the development of new chiral scaffolds for phosphoric acid based catalysis.

This work expands the range of chiral synthetic equivalents of cyanoethyl phosphorodiamidite $\mathbf{1}$ to $\beta$-hydroxyesters derivatives. It highlights phosphorodiamidites $4,8,9$ and 10 as chiral auxiliaries with potentially broad scope in different fields and applications, including chemical biology where the achiral analogue $\mathbf{1}$ is a wellknown privileged synthetic tool.

\section{Experimental Section}

Synthesis of the chiral phosphorodiamidites 4, 8, 9, 10 .

(1S)-2-cyanoethyl-1-phenyl $\quad N, N, N^{\prime}, N^{\prime}$-tetra-isopropylphosphorodiamidite $(S)-4$ has been prepared as reported previously. ${ }^{[3 b]}$

(R)-ethyl 3-((bis(diisopropylamino)phosphino)oxy)-4-cyanobutanoate $(\boldsymbol{R})$-8. To a suspension of bis(diisopropylamino)chlorophosphine $(850 \mathrm{mg}$, $3.18 \mathrm{mmol})$ in dry ether $(10 \mathrm{~mL})$ at $0{ }^{\circ} \mathrm{C}$ was added dropwise a solution of ethyl $(R)$-4-cyano-3-hydroxybutyrate $(500 \mathrm{mg}, 3.18 \mathrm{mmol})$ and triethylamine $(488 \mu \mathrm{L}, 3.50 \mathrm{mmol})$ in dry ether $(5 \mathrm{~mL})$. The mixture was allowed to warm to room temperature and stirred overnight. The white solid was filtered off, the solution was evaporated under reduced pressure to afford the desired phosphorodiamidite $(\boldsymbol{R})-\mathbf{8}(1.05 \mathrm{~g}, 85 \%) .{ }^{31} \mathrm{P}$ NMR $(202.5 \mathrm{~Hz}$, $\left.\mathrm{CDCl}_{3}\right): \delta=113.0 \mathrm{ppm} ;{ }^{1} \mathrm{H}$ NMR $\left(500.1 \mathrm{MHz}, \mathrm{CDCl}_{3}\right): \delta=4.22-4.17(\mathrm{~m}$, $1 \mathrm{H}, \mathrm{OCH}), 4.15-4.10\left(\mathrm{~m}, 2 \mathrm{H}, \mathrm{OCH}_{2}\right), 3.55-3.47(\mathrm{~m}, 4 \mathrm{H}, \mathrm{NCH}), 2.86(\mathrm{~d}, J=$ $15.9,4.8 \mathrm{~Hz}, 2 \mathrm{H}), 2.77(\mathrm{dd}, J=15.9,4.5 \mathrm{~Hz}, 1 \mathrm{H}), 2.70(\mathrm{dd}, J=15.9,8.4 \mathrm{~Hz}$, $1 \mathrm{H}), 1.25(\mathrm{t}, J=7.0 \mathrm{~Hz}, 3 \mathrm{H}, \mathrm{Me}), 1.17-1.15\left(24 \mathrm{H}, \mathrm{CHMe}_{2}\right) ;{ }^{13} \mathrm{C}$ NMR $(75.5$ $\left.\mathrm{MHz}, \mathrm{CDCl}_{3}\right): \delta=170.1(\mathrm{CO}), 116.9(\mathrm{CN}), 65.6(\mathrm{~d}, J=18.9 \mathrm{~Hz}, \mathrm{OCH}), 60.5$ $\left(\mathrm{CH}_{2}\right), 44.8(\mathrm{NCH}), 44.6(\mathrm{NCH}), 39.5\left(\mathrm{CH}_{2}\right), 39.4\left(\mathrm{CH}_{2}\right), 24.3\left(\mathrm{CH}_{3}\right), 24.1$ $\left(\mathrm{CH}_{3}\right), 24.0\left(\mathrm{CH}_{3}\right), 14.0\left(\mathrm{CH}_{3}\right)$; IR : $v_{\max }=2969,2932,2872,1733,1459,1392$, calcd. for $\mathrm{C}_{19} \mathrm{H}_{41} \mathrm{~N}_{3} \mathrm{O}_{4} \mathrm{P}\left[\mathrm{M}+\mathrm{H}_{2} \mathrm{O}+\mathrm{H}\right]^{+}$, 406.2835. Found, 406.2831. $[\alpha] \mathrm{D}=$ $-16\left(c=1.5, \mathrm{CHCl}_{3}\right)$.

(S)-Diethyl malate $N, N, N$, $N$ '-tetraisopropylphosphorodiamidite, (S)-9. Phosphorodiamidite $(\boldsymbol{S})-\mathbf{9}$ was obtained in $92 \%$ yield $(1.02 \mathrm{~g})$ from $0.70 \mathrm{~g}$ (2.6 mmol) of bis(diisopropylamino)chlorophosphine and $500 \mathrm{mg}$ of L-(-)malate $(2.6 \mathrm{mmol}) .{ }^{31} \mathrm{P}$ NMR $\left(202.5 \mathrm{~Hz}, \mathrm{CDCl}_{3}\right): \delta=118.9 \mathrm{ppm} ;{ }^{1} \mathrm{H}$ NMR $\left(500.1 \mathrm{MHz}, \mathrm{CDCl}_{3}\right): \delta 4.52-4.44(\mathrm{~m}, 1 \mathrm{H}, \mathrm{OCH}), 4.19(\mathrm{q}, J=7.2 \mathrm{~Hz}, 2 \mathrm{H}$, $\mathrm{OCH}_{2}$ ), 4.12 (q, $J=7.2 \mathrm{~Hz}, 2 \mathrm{H}, \mathrm{OCH}_{2}$ ), 3.60-3.47 (m, 4H, NCH), 2.87 (dd, $J=15.2,6.1 \mathrm{~Hz}, 1 \mathrm{H}), 2.80(\mathrm{dd}, J=15.2,6.6 \mathrm{~Hz}, 1 \mathrm{H}), 1.29-1.21(\mathrm{~m}, 6 \mathrm{H}, \mathrm{Me})$, 1.17 (s, $12 \mathrm{H}, \mathrm{CHMe} 2), 1.15$ (s, $12 \mathrm{H}, \mathrm{CHMe} 2) ;{ }^{13} \mathrm{C} \mathrm{NMR}\left(75.5 \mathrm{MHz}, \mathrm{CDCl}_{3}\right)$ : $\delta=172.1(\mathrm{CO}), 170.3(\mathrm{CO}), 69.6(\mathrm{~d}, J=18.2 \mathrm{~Hz}, \mathrm{OCH}), 61.0\left(\mathrm{CH}_{2}\right), 60.7$ $\left(\mathrm{CH}_{2}\right), 45.1(\mathrm{NCH}), 44.9(\mathrm{NCH}), 39.5\left(\mathrm{~d}, J=4.6 \mathrm{~Hz}, \mathrm{CH}_{2}\right), 24.5\left(\mathrm{CH}_{3}\right), 24.4$ $\left(\mathrm{CH}_{3}\right), 24.3\left(\mathrm{CH}_{3}\right), 14.3\left(\mathrm{CH}_{3}\right), 14.2\left(\mathrm{CH}_{3}\right)$; IR : $v_{\max }=2970,2934,2873,1737$, 1464, 1369, 1245, 1180, 1157, 1116, 1095, 1024, 1024, 953, 867; HR-MS (ESI): $m / z$ calcd. for $\mathrm{C}_{20} \mathrm{H}_{44} \mathrm{~N}_{2} \mathrm{O}_{6} \mathrm{P}\left[\mathrm{M}+\mathrm{H}_{2} \mathrm{O}+\mathrm{H}\right]^{+}$, 439.2937. Found, 439.2939. $[\alpha]_{\mathrm{D}}=-4.5\left(c=0.5, \mathrm{CHCl}_{3}\right)$

$N$-(tert-Butoxycarbonyl)-L-threonine methyl ester $N, N, N^{\prime}, N^{\prime}$ tetraisopropylphosphorodiamidite, $(2 S, 3 R)-10$. Phosphorodiamidite $(2 S, 3 R)-10$ was obtained quantitative yield $(3.5 \mathrm{~g})$ from $2.0 \mathrm{~g}$ of bis(diisopropylamino)chlorophosphine $\quad(7.55 \mathrm{mmol})$ and $\mathrm{N}$-(tertbutoxycarbonyl)-L-threonine methyl ester $(1.76 \mathrm{~g}, 7.55 \mathrm{mmol}) .{ }^{31} \mathrm{P}$ NMR $\left(202.5 \mathrm{~Hz}, \mathrm{CDCl}_{3}\right): \delta=111.4 \mathrm{ppm} ;{ }^{1} \mathrm{H}$ NMR $\left(500.1 \mathrm{MHz}, \mathrm{CDCl}_{3}\right): \delta=5.22$ $(\mathrm{d}, J=9.6 \mathrm{~Hz}, 1 \mathrm{H}), 4.37-4.32(\mathrm{~m}, 1 \mathrm{H}, \mathrm{OCH}), 4.21(\mathrm{~d}, J=9.6 \mathrm{~Hz}, 1 \mathrm{H}), 3.71$ (s, 3H, Me), 3.53-3.40 (m, 4H, NCH), 1.45 (s, 9H, $\mathrm{CMe}_{3}$ ), 1.32 (d, $J=6.6$ $\mathrm{Hz}, 3 \mathrm{H}, \mathrm{Me}), 1.18-1.15$ (m, 24H, CHMe 2$) ;{ }^{13} \mathrm{C} \mathrm{NMR}\left(75.5 \mathrm{MHz}, \mathrm{CDCl}_{3}\right): \delta$ $=171.5(\mathrm{CO}), 156.1(\mathrm{CO}), 79.5\left(\mathrm{CMe}_{3}\right), 69.9(\mathrm{~d}, J=16.6 \mathrm{~Hz}, \mathrm{OCH}), 59.0(\mathrm{~d}$, $J=4.1 \mathrm{~Hz}, \mathrm{CH}), 52.0\left(\mathrm{OCH}_{3}\right), 44.9(\mathrm{NCH}), 44.7(\mathrm{NCH}), 44.5(\mathrm{NCH})$ $28.3\left(\mathrm{CMe}_{3}\right), 24.5\left(\mathrm{CH}_{3}\right), 24.4\left(\mathrm{CH}_{3}\right), 24.2\left(\mathrm{CH}_{3}\right), 24.1\left(\mathrm{CH}_{3}\right), 19.3(\mathrm{~d}$, $\left.J=8.1 \mathrm{~Hz}, \mathrm{CH}_{3}\right) ; \mathrm{IR}: v_{\max }=3447,2968,2930,1758,1717,1485,1460,1362$, $1318,1183,1156,1118,1087,1069,1024,1002,953,902,866$; HR-MS (ESI): $m / z$ calcd. for $\mathrm{C}_{22} \mathrm{H}_{49} \mathrm{~N}_{3} \mathrm{O}_{6} \mathrm{P}\left[\mathrm{M}+\mathrm{H}_{2} \mathrm{O}+\mathrm{H}\right]^{+}$, 482.3359. Found, 482.3344. $[\alpha]_{\mathrm{D}}^{20}=+7.0\left(c=1.5, \mathrm{CHCl}_{3}\right)$

Representative procedure for the synthesis of phosphates 6 (Scheme 2 and Scheme 3). A solution of (1S)-2-cyanoethyl-1-phenyl $N, N, N^{\prime}, N^{\prime}$-tetraisopropylphosphorodiamidite $(S)-4(180 \mathrm{mg}, 0.48 \mathrm{mmol})$ in anhydrous $1,2-$ dichloroethane $(5 \mathrm{~mL})$ was added dropwise at r.t. to a solution of $\mathrm{rac}$-BINOL $(114 \mathrm{mg}, 0.40 \mathrm{mmol})$ and $1 H$-tetrazole $(117 \mathrm{mg}, 1.67 \mathrm{mmol})$ in 1,2 dichloroethane $(20 \mathrm{~mL})$. The mixture was heated at $80{ }^{\circ} \mathrm{C}$ for $3 \mathrm{~h}$. The reaction was quenched by addition of saturated aqueous $\mathrm{NaHCO}_{3}(20 \mathrm{~mL})$ and the mixture was extracted twice with DCM $(2 \times 20 \mathrm{~mL})$. The organic phase was dried over $\mathrm{MgSO}_{4}$ and the solvents were removed under reduced 1378, 1363, 1310, 1183, 1116, 1055, 1023, 954, 867, 708; HR-MS (ESI): $m / z$ 
pressure. The crude phosphites (1:1 mixture of diastereomers, $\delta{ }^{31} \mathrm{P}=146.7$ and $135.2 \mathrm{ppm})$ were engaged in the next step without further purification. Phosphites were dissolved in DCM ( $1 \mathrm{~mL})$. TBHP $(218 \mu \mathrm{L}, 5.5 \mathrm{M}$ in decane, $1.20 \mathrm{mmol}$ ) was added to the solution at $0{ }^{\circ} \mathrm{C}$. Then the reaction mixture was allowed to warm to room temperature and stirred for 30 minutes. After addition of saturated aqueous $\mathrm{Na}_{2} \mathrm{~S}_{2} \mathrm{O}_{3}$, the layers were separated, the organic layer was dried over $\mathrm{MgSO}_{4}$ and concentrated in vacuo. The crude mixture was purified by chromatography on silica gel (heptane/EtOAc $=6: 4$ to $4: 6$ ) to afford $120 \mathrm{mg}$ of $\mathbf{6 a , a}$ '(63\% yield). Phosphates $\mathbf{6 a}$ and $\mathbf{6 a}$ ' were separated by semi-preparative HPLC on a $\mathrm{SiO}_{2}$ column $(250$ x $10 \mathrm{~mm}, 5 \mathrm{mic})$ with a heptane/EtOAc gradient (8:2 to $4: 6)$. 6a: retention time $16.7 \mathrm{~min}, 40 \mathrm{mg}$, $33 \%$ yield; 6a': retention time $17.7 \mathrm{~min}, 48 \mathrm{mg}, 40 \%$ yield.

6a: ${ }^{31} \mathrm{P}$ NMR $\left(202.5 \mathrm{~Hz}, \mathrm{CDCl}_{3}\right): \delta=1.5 \mathrm{ppm} ;{ }^{1} \mathrm{H} \mathrm{NMR}\left(500 \mathrm{MHz}, \mathrm{CDCl}_{3}\right.$ ) $\delta=8.04(\mathrm{~d}, J=8.9 \mathrm{~Hz}, 1 \mathrm{H}), 7.99(\mathrm{~d}, J=9.1 \mathrm{~Hz}, 1 \mathrm{H}), 7.97(\mathrm{~d}, J=9.1 \mathrm{~Hz}, 1 \mathrm{H})$, $7.92(\mathrm{~d}, J=8.4 \mathrm{~Hz}, 1 \mathrm{H}), 7.62(\mathrm{~d}, J=8.6 \mathrm{~Hz}, 1 \mathrm{H}), 7.49(\mathrm{t}, J=6.6 \mathrm{~Hz}, 1 \mathrm{H})$, $7.46(\mathrm{~d}, J=8.6 \mathrm{~Hz}, 2 \mathrm{H}), 7.36-7.27(\mathrm{~m}, 9 \mathrm{H}), 5.89\left(\mathrm{ddd}, J_{\mathrm{H}, \mathrm{P}}=7.6 \mathrm{~Hz}, J=6.5\right.$, $5.3 \mathrm{~Hz}, 1 \mathrm{H}, \mathrm{OCH}), 3.10\left(\mathrm{dd}, J=16.9,5.3 \mathrm{~Hz}, 1 \mathrm{H}, \mathrm{CH}_{2} \mathrm{CN}\right), 3.01(\mathrm{dd}, J=$ $\left.16.9,6.5 \mathrm{~Hz}, 1 \mathrm{H}, \mathrm{CH}_{2} \mathrm{CN}\right) ;{ }^{13} \mathrm{C} \mathrm{NMR}\left(125.8 \mathrm{MHz} \mathrm{CDCl}_{3}\right): \delta=147.5\left(\mathrm{~d}, J_{C}\right.$ $\left.{ }_{P}=11.8 \mathrm{~Hz}, \mathrm{C}-\mathrm{O}\right), 146.2\left(\mathrm{~d}, J_{C-P}=8.2 \mathrm{~Hz}, \mathrm{C}-\mathrm{O}\right), 136.6\left(\mathrm{~d}, J_{C-P}=4.0 \mathrm{~Hz}, \mathrm{C}_{\mathrm{Ph}}\right)$, $132.4(\mathrm{C}), 132.2(\mathrm{C}), 132.0(\mathrm{C}), 131.7(\mathrm{CH}), 131.6(\mathrm{CH}), 129.8(\mathrm{CH}), 129.2$ $(\mathrm{CH}), 128.7(\mathrm{CH}), 127.4(\mathrm{CH}), 127.3(\mathrm{CH}), 127.1(\mathrm{CH}), 127.0(\mathrm{CH}), 126.2$ $(\mathrm{CH}), 126.1(\mathrm{CH}), 126.0(\mathrm{CH}), 121.6(\mathrm{C}), 121.2(\mathrm{C}), 120.6(\mathrm{CH}), 115.8(\mathrm{CN})$, $76.9\left(\mathrm{~d}, J_{C-P}=4.6 \mathrm{~Hz}, \mathrm{CH}, \mathrm{OCH}\right), 27.8\left(\mathrm{~d}, J_{C-P}=6.7 \mathrm{~Hz}, \mathrm{CH}_{2}\right)$; IR : $v_{\max }$ $=3064,3015,2927,2853,1620,1591,1508,1465,1434,1362,1296,1227$. 1010, 977, 951, 748, 697. HR-MS (ESI): $m / z$ calcd. for $\mathrm{C}_{29} \mathrm{H}_{21} \mathrm{NO}_{4} \mathrm{P}[\mathrm{M}+\mathrm{H}]^{+}$: 478.1208, Found: 478.1249; $[\alpha] \mathrm{D}=+296\left(c=1, \mathrm{CHCl}_{3}\right)$.

6a': ${ }^{31} \mathrm{P}$ NMR $\left(202.5 \mathrm{~Hz}, \mathrm{CDCl}_{3}\right): \delta=2.1 \mathrm{ppm} ;{ }^{1} \mathrm{H}$ NMR $(500.1 \mathrm{MHz}$, $\left.\mathrm{CDCl}_{3}\right): \delta=8.04(\mathrm{~d}, J=9.0 \mathrm{~Hz}, 1 \mathrm{H}), 7.94(\mathrm{~d}, J=8.3 \mathrm{~Hz}, 1 \mathrm{H}), 7.91(\mathrm{~d}, J=$ $8.3 \mathrm{~Hz}, 1 \mathrm{H}), 7.83(\mathrm{~d}, J=8.8 \mathrm{~Hz}, 1 \mathrm{H}), 7.61(\mathrm{~d}, J=8.9 \mathrm{~Hz}, 1 \mathrm{H}), 7.50-7.43$ (7H), $7.35(\mathrm{~d}, J=8.8 \mathrm{~Hz}, 1 \mathrm{H}), 7.31-7.25(3 \mathrm{H}), 6.76(\mathrm{~d}, J=8.8 \mathrm{~Hz}, 1 \mathrm{H}), 5.93$ $(\mathrm{m}, 1 \mathrm{H}, \mathrm{OCH}), 3.04\left(\mathrm{dd}, J=17.0,6.3 \mathrm{~Hz}, 1 \mathrm{H}, \mathrm{CH}_{2} \mathrm{CN}\right), 3.00(\mathrm{dd}, J=17.0$, $\left.6.3 \mathrm{~Hz}, 1 \mathrm{H}, \mathrm{CH}_{2} \mathrm{CN}\right) ;{ }^{13} \mathrm{C} \mathrm{NMR}\left(125.8 \mathrm{MHz} \mathrm{CDCl}_{3}\right): \delta=147.0\left(\mathrm{~d}, J_{C-P}=\right.$ $11.5 \mathrm{~Hz}, \mathrm{C}-\mathrm{O}), 146.0$ (d, $\left.J_{C-P}=7.7 \mathrm{~Hz}, \mathrm{C}-\mathrm{O}\right), 136.3\left(\mathrm{~d}, J_{C-P}=3.1 \mathrm{~Hz}, \mathrm{C}_{\mathrm{Ph}}\right.$ ), $132.2(\mathrm{C}), 132.0(\mathrm{C}), 131.7(\mathrm{C}), 131.6(\mathrm{CH}), 131.0(\mathrm{CH}), 130.1(\mathrm{CH}), 129.2$ $(\mathrm{CH}), 128.6(\mathrm{CH}), 128.4(\mathrm{CH}), 127.2(\mathrm{CH}), 127.0(\mathrm{CH}), 126.9(\mathrm{CH}), 126.6$ $(\mathrm{CH}), 126.0(\mathrm{CH}), 125.9(\mathrm{CH}), 121.3(\mathrm{C}), 121.1(\mathrm{C}), 120.5(\mathrm{CH}), 120.0(\mathrm{CH})$, $115.1(\mathrm{CN}), 76.1\left(\mathrm{~d}, J_{C-P}=3.7 \mathrm{~Hz}, \mathrm{OCH}\right), 27.1\left(\mathrm{~d}, J_{C-P}=6.5 \mathrm{~Hz}, \mathrm{CH}_{2} \mathrm{CN}\right)$; IR : $v_{\max }=3067,2928,2854,1620,1591,1508,1464,1294,1227,1009,976$, 951, 896, 816, 729. HR-MS ( 478.1208. Found, 478.1203, $[\alpha] \mathrm{D}=-329\left(c=1, \mathrm{CHCl}_{3}\right)$

Phosphates $\mathbf{6 b}$ and $\mathbf{6} \mathbf{b}^{6}$ have been obtained via the same procedure $(50 \%$ yield). They were separated by semi-preparative HPLC on a $\mathrm{SiO}_{2}$ column $(250 \times 10 \mathrm{~mm}, 5 \mathrm{mic})$ with a heptane/EtOAc gradient $(8: 2$ to $4: 6)$. 6b: retention time $9.6 \mathrm{~min}, 43 \%$ yield; $6 \mathbf{b}$ ': retention time $10.5 \mathrm{~min}, 40 \%$ yield. 6b: ${ }^{31} \mathrm{P}$ NMR $\left(202.5 \mathrm{~Hz}, \mathrm{CDCl}_{3}\right): \delta=1.9 \mathrm{ppm} ;{ }^{1} \mathrm{H}$ NMR $(500.1 \mathrm{MHz}$, $\left.\mathrm{CDCl}_{3}\right): \delta=8.45(\mathrm{~d}, J=8.3 \mathrm{~Hz}, 1 \mathrm{H}), 7.82(\mathrm{~d}, J=8.2 \mathrm{~Hz}, 1 \mathrm{H}), 7.78(\mathrm{~d}, J=$ $8.2 \mathrm{~Hz}, 1 \mathrm{H}), 7.74(\mathrm{~d}, J=8.3 \mathrm{~Hz}, 1 \mathrm{H}), 7.68(\mathrm{t}, J=7.6 \mathrm{~Hz}, 1 \mathrm{H}), 7.60(\mathrm{t}, J=$ $7.4 \mathrm{~Hz}, 1 \mathrm{H}), 7.50-7.48(\mathrm{~m}, 3 \mathrm{H}), 7.43(\mathrm{t}, J=7.4 \mathrm{~Hz}, 1 \mathrm{H}), 7.37-7.34(\mathrm{~m}, 2 \mathrm{H})$, $7.28(\mathrm{~d}, J=7.3 \mathrm{~Hz}, 2 \mathrm{H}), 7.20(\mathrm{t}, J=7.4 \mathrm{~Hz}, 1 \mathrm{H}), 7.11-7.05(\mathrm{~m}, 2 \mathrm{H}), 6.93(\mathrm{t}$, $J=7.5 \mathrm{~Hz}, 2 \mathrm{H}), 6.89(\mathrm{t}, J=7.5 \mathrm{~Hz}, 2 \mathrm{H}), 6.47(\mathrm{~d}, J=7.5 \mathrm{~Hz}, 2 \mathrm{H}), 6.43(\mathrm{~d}, J$ $=7.5 \mathrm{~Hz}, 2 \mathrm{H}), 6.11\left(\mathrm{ddd}, J_{\mathrm{H}-\mathrm{P}}=9.0 \mathrm{~Hz}, J=7.2,5.5 \mathrm{~Hz}, 1 \mathrm{H}, \mathrm{OCH}\right), 3.05(\mathrm{dd}$, $\left.J=16.9,7.2 \mathrm{~Hz}, 1 \mathrm{H}, \mathrm{CH}_{2} \mathrm{CN}\right), 2.96\left(\mathrm{dd}, J=16.9,5.5 \mathrm{~Hz}, 1 \mathrm{H}, \mathrm{CH}_{2} \mathrm{CN}\right) ;{ }^{13} \mathrm{C}$ NMR $\left(125.8 \mathrm{MHz}, \mathrm{CDCl}_{3}\right): \delta=145.8\left(\mathrm{~d}, J_{C-P}=11.8 \mathrm{~Hz}, \mathrm{C}-\mathrm{O}\right), 144.8\left(\mathrm{~d}, J_{C}\right.$ $\left.{ }_{P}=8.2 \mathrm{~Hz}, \mathrm{C}-\mathrm{O}\right), 140.3(\mathrm{C}), 140.1(\mathrm{C}), 139.9(\mathrm{~d}, J=3.4 \mathrm{~Hz}, \mathrm{C}), 136.4(\mathrm{~d}, J$ $=4.4 \mathrm{~Hz}, \mathrm{C}), 134.5(\mathrm{C}), 134.3(\mathrm{C}), 130.0(\mathrm{CH}), 129.2(\mathrm{CH}), 129.1(\mathrm{CH})$, $128.1(\mathrm{CH}), 127.9(\mathrm{CH}), 127.9(\mathrm{CH}), 127.8(\mathrm{CH}), 127.7(\mathrm{CH}), 127.4(\mathrm{CH})$, $127.0(\mathrm{CH}), 126.9(\mathrm{CH}), 126.8(\mathrm{CH}), 126.6(\mathrm{CH}), 125.9(\mathrm{C}), 125.7(\mathrm{C}), 125.1$ $(\mathrm{C}), 122.7(\mathrm{CH}), 122.6(\mathrm{C}), 122.3(\mathrm{C}), 122.2(\mathrm{CH}), 115.6(\mathrm{CN}), 77.4\left(\mathrm{~d}, J_{C-P}\right.$ $=4.5 \mathrm{~Hz}, \mathrm{OCH}), 27.5\left(\mathrm{~d}, J_{C-P}=4.5 \mathrm{~Hz}, \mathrm{CH}_{2} \mathrm{CN}\right)$; IR : $v_{\max }=3058,3022,2959$, 2927, 1593, 1566, 1489, 1362, 1289, 1009, 1000, 946, 760; HR-MS (ESI): $m / z$ calcd. for $\mathrm{C}_{41} \mathrm{H}_{29} \mathrm{NO}_{4} \mathrm{P}[\mathrm{M}+\mathrm{H}]^{+}, 630.1834$. Found, 630.1829. $[\alpha]_{\mathrm{D}}^{20}=-$ $177\left(c=1, \mathrm{CHCl}_{3}\right)$. 6b': ${ }^{31} \mathrm{P}$ NMR $\left(202.5 \mathrm{~Hz}, \mathrm{CDCl}_{3}\right): \delta=1.4 \mathrm{ppm} ;{ }^{1} \mathrm{H}$ NMR $(500.1 \mathrm{MHz}$, $\left.\mathrm{CDCl}_{3}\right): \delta=8.44(\mathrm{~d}, J=8.2 \mathrm{~Hz}, 1 \mathrm{H}), 7.84(\mathrm{~d}, J=8.2 \mathrm{~Hz}, 1 \mathrm{H}), 7.77(\mathrm{~d}, J=$ $8.2 \mathrm{~Hz}, 2 \mathrm{H}), 7.67(\mathrm{t}, J=7.9 \mathrm{~Hz}, 1 \mathrm{H}), 7.61(\mathrm{t}, J=7.9 \mathrm{~Hz}, 1 \mathrm{H}), 7.55-7.47(\mathrm{~m}$, 4H), 7.39-7.32 (m, 5H), 7.10-7.06 (m, 2H), $6.90(\mathrm{t}, J=7.9 \mathrm{~Hz}, 4 \mathrm{H}), 6.44(\mathrm{~d}$, $J=7.9 \mathrm{~Hz}, 2 \mathrm{H}), 6.41(\mathrm{~d}, J=7.9 \mathrm{~Hz}, 2 \mathrm{H}), 5.99\left(\mathrm{ddd}, J=7.0,6.3 \mathrm{~Hz}, J_{\mathrm{H}, \mathrm{P}}=\right.$ $6.3 \mathrm{~Hz}, 1 \mathrm{H}), 3.04(\mathrm{dd}, J=16.7,7.0 \mathrm{~Hz}, 1 \mathrm{H}) ; 2.97\left(\mathrm{ddd}, J=16.7,6.3 \mathrm{~Hz}, J_{\mathrm{H}, \mathrm{P}}\right.$ $=1.9 \mathrm{~Hz}, 1 \mathrm{H}) ;{ }^{13} \mathrm{C} \mathrm{NMR}\left(125.8 \mathrm{MHz}, \mathrm{CDCl}_{3}\right): \delta=146.0(\mathrm{~d}, J=11.4 \mathrm{~Hz}$, C), $144.8\left(\mathrm{~d}, J_{C-P}=9.0 \mathrm{~Hz}, \mathrm{C}\right), 140.2\left(\mathrm{~d}, J_{C-P}=5.6 \mathrm{~Hz}, \mathrm{C}\right), 140.0(\mathrm{C}), 139.8$ (C), $136.4(\mathrm{C}), 134.5(\mathrm{C}), 134.4(\mathrm{C}), 130.1(\mathrm{CH}), 129.3(\mathrm{CH}), 129.2(\mathrm{CH})$ $129.1(\mathrm{CH}), 128.1(\mathrm{CH}), 127.9(\mathrm{CH}), 127.6(\mathrm{CH}), 127.4(\mathrm{CH}), 126.9(\mathrm{CH})$, $126.7(\mathrm{CH}), 125.7(\mathrm{C}), 125.4(\mathrm{C}), 122.6(\mathrm{CH}), 122.3(\mathrm{CH}), 115.6(\mathrm{CN}), 77.9$ $\left(\mathrm{d}, J_{C-P}=5.5 \mathrm{~Hz}, \mathrm{OCH}\right), 27.3\left(\mathrm{~d}, J_{C-P}=9.7 \mathrm{~Hz}, \mathrm{CH}_{2} \mathrm{CN}\right)$; IR: $v_{\max }=3057$, 2927, 2855, 1633, 1593, 1566, 1488, 1362, 1287, 998, 945, 906, 761; HRMS (ESI): $m / z$ calcd. for $\mathrm{C}_{41} \mathrm{H}_{29} \mathrm{NO}_{4} \mathrm{P}[\mathrm{M}+\mathrm{H}]^{+}, 630.1834$. Found, 630.1863, $[\alpha]_{\mathrm{D}}^{20}=+96\left(c=1, \mathrm{CHCl}_{3}\right)$

Phosphates $\mathbf{6 c}$ and $\mathbf{6} \mathbf{c}^{\mathbf{6}}$ have been obtained via the same procedure (42\% yield). They were separated by semi-preparative HPLC on a $\mathrm{SiO}_{2}$ column $(250 \times 10 \mathrm{~mm}, 5 \mathrm{mic})$ with a heptane/EtOAc gradient $(8: 2$ to $5: 5)$. 6c: retention time $11.1 \mathrm{~min}, 50 \%$ yield; $6 \mathbf{c}$ ': retention time $12.0 \mathrm{~min}, 40 \%$ yield 6c: ${ }^{31} \mathrm{P}$ NMR $\left(202.5 \mathrm{~Hz}, \mathrm{CDCl}_{3}\right): \delta=-3.0 \mathrm{ppm} ;{ }^{1} \mathrm{H}$ NMR $(500.1 \mathrm{MHz}$ $\left.\mathrm{CDCl}_{3}\right): \delta=9.42-9.38(\mathrm{~m}, 2 \mathrm{H}), 7.95(\mathrm{~d}, J=7.7 \mathrm{~Hz}, 1 \mathrm{H}), 7.83(\mathrm{~d}, J=8.6 \mathrm{~Hz}$, $1 \mathrm{H}), 7.78(\mathrm{~d}, J=7.6 \mathrm{~Hz}, 1 \mathrm{H}), 7.72-7.68(\mathrm{~m}, 4 \mathrm{H}), 7.64(\mathrm{~d}, J=8.6 \mathrm{~Hz}, 1 \mathrm{H})$, $7.59(\mathrm{~s}, 2 \mathrm{H}), 7.56-7.51(\mathrm{~m}, 2 \mathrm{H}), 7.11(\mathrm{t}, J=7.6 \mathrm{~Hz}, 2 \mathrm{H}), 7.07(\mathrm{~d}, J=7.6 \mathrm{~Hz}$ $1 \mathrm{H}), 6.99-6.90(\mathrm{~m}, 6 \mathrm{H}), 6.70(\mathrm{~d}, J=7.6 \mathrm{~Hz}, 2 \mathrm{H}), 6.51(\mathrm{t}, J=7.0 \mathrm{~Hz}, 4 \mathrm{H})$, $5.51\left(\mathrm{ddd}, J_{\mathrm{H}, \mathrm{P}}=7.7 \mathrm{~Hz}, J=6.7,4.7 \mathrm{~Hz}, 1 \mathrm{H}, \mathrm{OCH}\right), 2.97(\mathrm{dd}, J=16.8,6.7$ $\mathrm{Hz}, 1 \mathrm{H}, \mathrm{CH}_{2} \mathrm{CN}$ ); 2.65 (dd, $\left.J=16.8,4.7 \mathrm{~Hz}, 1 \mathrm{H}, \mathrm{CH}_{2} \mathrm{CN}\right) ;{ }^{13} \mathrm{C} \mathrm{NMR}(125.8$ $\left.\mathrm{MHz}_{\mathrm{CDCl}}\right): \delta=147.6\left(\mathrm{~d}, J_{C-P}=11.1 \mathrm{~Hz}, \mathrm{C}\right), 146.6\left(\mathrm{~d}, J_{C-P}=8.1 \mathrm{~Hz}, \mathrm{C}\right)$, 141.6 (C), 141.3 (C), 139.4 (d, $J=3.3 \mathrm{~Hz}, \mathrm{C}), 135.7$ (d, $J=6.0 \mathrm{~Hz}, \mathrm{C}), 135.1$ (C), $134.7(\mathrm{C}), 133.6(\mathrm{C}), 133.3(\mathrm{C}), 129.8(\mathrm{CH}), 129.6(\mathrm{CH}), 129.5(\mathrm{CH})$, $129.1(\mathrm{CH}), 129.0(\mathrm{C}), 128.6(\mathrm{CH}), 128.5(\mathrm{CH}), 128.3(\mathrm{CH}), 128.0(\mathrm{CH})$, $127.9(\mathrm{CH}), 127.8(\mathrm{CH}), 127.7(\mathrm{CH}), 127.5(\mathrm{CH}), 127.4(\mathrm{CH}), 127.1(\mathrm{CH})$, 127.0 (CH), $126.7(\mathrm{CH}), 126.6(\mathrm{CH}), 125.7(\mathrm{C}), 125.5(\mathrm{C}), 125.2(\mathrm{CH}), 122.0$ (C), $121.4(\mathrm{C}), 115.1(\mathrm{CN}), 76.9\left(\mathrm{~d}, J_{C-P}=5.3 \mathrm{~Hz}, \mathrm{OCH}\right), 27.7\left(\mathrm{~d}, J_{C-P}=4.6\right.$ $\mathrm{Hz}, \mathrm{CH}_{2} \mathrm{CN}$ ); IR : $v_{\max }=3056,2926,1598,1487,1456,1425,1388,1300$, 1232, 1124, 1016, 914, 890, 869, 751; HR-MS (ESI) $\mathrm{m} / \mathrm{z}$ calcd. for $\mathrm{C}_{49} \mathrm{H}_{33} \mathrm{NO}_{4} \mathrm{P}[\mathrm{M}+\mathrm{H}]^{+}, 730.2147$. Found, 730.2153; $[\alpha] \mathrm{D}=-367(c=1.5$, $\mathrm{CHCl}_{3}$ ).

6c': ${ }^{31} \mathrm{P}$ NMR $\left(202.5 \mathrm{~Hz}, \mathrm{CDCl}_{3}\right): \delta=-2.2 \mathrm{ppm} ;{ }^{1} \mathrm{H}$ NMR $(500.1 \mathrm{MHz}$, $\left.\mathrm{CDCl}_{3}\right): \delta=9.67(\mathrm{~d}, J=8.6 \mathrm{~Hz}, 1 \mathrm{H}), 8.89(\mathrm{~d}, J=8.7 \mathrm{~Hz}, 1 \mathrm{H}), 8.03(\mathrm{~d}, J=$ $8.0 \mathrm{~Hz}, 1 \mathrm{H}), 7.89-7.84(\mathrm{~m}, 3 \mathrm{H}), 7.78-7.74(\mathrm{~m}, 3 \mathrm{H}), 7.65(\mathrm{~d}, J=8.7 \mathrm{~Hz}, 1 \mathrm{H})$, $7.61(\mathrm{~s}, 1 \mathrm{H}), 7.57(\mathrm{~s}, 1 \mathrm{H}), 7.53(\mathrm{t}, J=7.6 \mathrm{~Hz}, 1 \mathrm{H}), 7.47-7.39(\mathrm{~m}, 5 \mathrm{H}), 7.12$ $7.08(\mathrm{~m}, 3 \mathrm{H}), 6.95-6.91(\mathrm{~m}, 4 \mathrm{H}), 6.55(\mathrm{~d}, J=8.6 \mathrm{~Hz}, 2 \mathrm{H}), 6.46(\mathrm{~d}, J=8.0$ $\mathrm{Hz}, 2 \mathrm{H}), 5.85\left(\mathrm{ddd}, J_{\mathrm{H}, \mathrm{P}}=8.0 \mathrm{~Hz}, J=8.0,3.8 \mathrm{~Hz}, 1 \mathrm{H}, \mathrm{OCH}\right), 2.14(\mathrm{dd}, J=$ $\left.16.5,8.0 \mathrm{~Hz}, 1 \mathrm{H}, \mathrm{CH}_{2} \mathrm{CN}\right), 2.02\left(\mathrm{dd}, J=16.5,3.8 \mathrm{~Hz}, 1 \mathrm{H}, \mathrm{CH}_{2} \mathrm{CN}\right) ;{ }^{13} \mathrm{C} \mathrm{NMR}$ $\left(125.8 \mathrm{MHz}, \mathrm{CDCl}_{3}\right): \delta=147.6(\mathrm{~d}, J=11.1 \mathrm{~Hz}, \mathrm{C}), 146.8(\mathrm{~d}, J=8.4 \mathrm{~Hz}, \mathrm{C})$, 141.7 (C), 141.4 (C), 139.5 (d, $J=3.1 \mathrm{~Hz}, \mathrm{C}), 137.0$ (C), 135.0 (C), 134.9 (C), $133.7(\mathrm{C}), 133.5(\mathrm{C}), 130.0(\mathrm{CH}), 129.7(\mathrm{CH}), 129.6(\mathrm{CH}), 129.5(\mathrm{CH})$ $129.4(\mathrm{CH}), 129.3(\mathrm{CH}), 129.0(\mathrm{CH}), 128.8(\mathrm{C}), 128.5(\mathrm{CH}), 127.9(\mathrm{CH})$ $127.8(\mathrm{CH}), 127.7(\mathrm{CH}), 127.6(\mathrm{CH}), 127.4(\mathrm{CH}), 127.3(\mathrm{CH}), 127.2(\mathrm{CH})$ $127.1(\mathrm{CH}), 127.0(\mathrm{CH}), 126.7(\mathrm{CH}), 126.3(\mathrm{CH}), 126.0(\mathrm{C}), 125.5(\mathrm{C}), 121.9$ (C), $121.5(\mathrm{C}), 114.7(\mathrm{CN}), 76.9$ (d, $J=4.3 \mathrm{~Hz}, \mathrm{OCH}), 26.5(\mathrm{~d}, J=10.9 \mathrm{~Hz}$, $\mathrm{CH}_{2} \mathrm{CN}$ ); IR : $v_{\max }=3059,2926,2854,1598,1558,1456,1387,1300,1231$, 1023, 1010, 914, 751; HR-MS (ESI): $\mathrm{m} / z$ calcd. for $\mathrm{C}_{49} \mathrm{H}_{33} \mathrm{NO}_{4} \mathrm{P}[\mathrm{M}+\mathrm{H}]^{+}$ 730.2147. Found, 730.2151; $[\alpha] \mathrm{D}=+303\left(c=1.5, \mathrm{CHCl}_{3}\right)$.

Phosphates $\mathbf{6 d}$ and $\mathbf{6 d}$ ' have been obtained via the same procedure ( $88 \%$ total yield). They were separated on a Combiflash ${ }^{\circledR}$ system by using a silica gel column, with a toluene/THF gradient (99:1 to $94: 6)$ as the eluent. 6d: $\mathbf{R}_{\mathrm{f}}=$ 0.2 (toluene/THF $=90: 10), 48 \%$ yield; $6 \mathbf{d}$ ': $\mathrm{R}_{\mathrm{f}}=0.17,40 \%$ yield

6d: ${ }^{31} \mathrm{P}$ NMR $\left(202.5 \mathrm{~Hz}, \mathrm{CDCl}_{3}\right): \delta=-10.7 \mathrm{ppm} ;{ }^{1} \mathrm{H}$ NMR $(500.1 \mathrm{MHz}$, $\left.\mathrm{CDCl}_{3}\right): \delta=7.46-7.44(\mathrm{~m}, 3 \mathrm{H}), 7.41-7.38(\mathrm{~m}, 2 \mathrm{H}), 7.26(\mathrm{t}, J=7.4 \mathrm{~Hz}, 1 \mathrm{H})$, $7.17(\mathrm{~d}, J=7.4 \mathrm{~Hz}, 1 \mathrm{H}), 7.12(\mathrm{t}, J=7.4 \mathrm{~Hz}, 2 \mathrm{H}), 7.03(\mathrm{t}, J=7.4 \mathrm{~Hz}, 1 \mathrm{H})$, 
$6.47(\mathrm{~d}, J=7.4 \mathrm{~Hz}, 1 \mathrm{H}), 5.81\left(\mathrm{dt}, J_{\mathrm{H}, \mathrm{P}}=8.2 \mathrm{~Hz}, J=5.5 \mathrm{~Hz}, 1 \mathrm{H}, \mathrm{OCH}\right), 3.14$ $3.06(\mathrm{~m}, 2 \mathrm{H}), 2.96(\mathrm{~d}, J=5.5 \mathrm{~Hz}, 2 \mathrm{H}), 2.90-2.81(\mathrm{~m}, 2 \mathrm{H}), 2.31-2.24(\mathrm{~m}, 2 \mathrm{H})$, 2.08-1.98 (m, 2H); ${ }^{13} \mathrm{C}$ NMR (75.5 MHz, $\left.\mathrm{CDCl}_{3}\right): \delta=146.8(\mathrm{C}), 146.6(\mathrm{C})$, 145.8 (d, $J=10.3 \mathrm{~Hz}, \mathrm{O}-\mathrm{C}), 144.3$ (d, $J=7.1 \mathrm{~Hz}, \mathrm{O}-\mathrm{C}), 139.3$ (d, $J=3.6 \mathrm{~Hz}$, C), $138.8(\mathrm{C}), 136.3\left(\mathrm{~d}, J=5.7 \mathrm{~Hz}, \mathrm{C}_{\mathrm{Ph}}\right), 129.8(\mathrm{CH}), 129.0(\mathrm{CH}), 128.4(\mathrm{CH})$ $126.4(\mathrm{CH}), 123.4(\mathrm{CH}), 123.0(\mathrm{CH}), 121.5(\mathrm{CH}), 121.0(\mathrm{CH}), 115.3(\mathrm{CN})$, $76.1(\mathrm{~d}, J=4.8 \mathrm{~Hz}, \mathrm{OCH}), 59.2(\mathrm{C}), 38.5\left(\mathrm{CH}_{2}\right), 38.2\left(\mathrm{CH}_{2}\right), 30.6\left(\mathrm{CH}_{2}\right), 27.3$ $\left(\mathrm{d}, J=5.0 \mathrm{~Hz}, \mathrm{CH}_{2} \mathrm{CN}\right)$; IR : $v_{\max }=3018,2956,2856,1616,1584,1466,1293$, 1222, 1159, 1052, 1013, 997, 931, 905; HR-MS (ESI): $\mathrm{m} / \mathrm{z}$ calcd. for $\mathrm{C}_{26} \mathrm{H}_{23} \mathrm{NO}_{4} \mathrm{P}[\mathrm{M}+\mathrm{H}]^{+}, 444.1365$. Found, 444.1356. $[\alpha]_{\mathrm{D}}^{20}=-183(c=1$, $\mathrm{CHCl}_{3}$ ).

6d': ${ }^{31} \mathrm{P}$ NMR $\left(202.5 \mathrm{~Hz}, \mathrm{CDCl}_{3}\right): \delta=-11.4 \mathrm{ppm} ;{ }^{1} \mathrm{H}$ NMR $(500.1 \mathrm{MHz}$, $\left.\mathrm{CDCl}_{3}\right): \delta=7.34-7.29(\mathrm{~m}, 3 \mathrm{H}), 7.26-7.10(\mathrm{~m}, 7 \mathrm{H}), 6.88(\mathrm{~d}, J=7.7 \mathrm{~Hz}, 1 \mathrm{H})$, $5.73\left(\mathrm{dt}, J_{\mathrm{H}, \mathrm{P}}=5.5 \mathrm{~Hz}, J=5.5 \mathrm{~Hz}, 1 \mathrm{H}\right), 3.10(\mathrm{dd}, J=16.9,5.5 \mathrm{~Hz}, 1 \mathrm{H}), 3.08$ $3.02(\mathrm{~m}, 2 \mathrm{H}), 2.98(\mathrm{dd}, J=16.9,5.5 \mathrm{~Hz}, 1 \mathrm{H}), 2.84-2.78(\mathrm{~m}, 2 \mathrm{H}), 2.25-2.20$ (m, 2H), 2.05-1.98 (m, 1H), 1.92-1.86 (m, 1H); ${ }^{13} \mathrm{C}$ NMR $(75.5 \mathrm{MHz}$, $\left.\mathrm{CDCl}_{3}\right): \delta=146.8(\mathrm{C}), 146.6(\mathrm{C}), 146.1$ (d, $\left.J=10.4 \mathrm{~Hz}, \mathrm{O}-\mathrm{C}\right), 144.4$ (d, $J=$ $7.1 \mathrm{~Hz}, \mathrm{O}-\mathrm{C}), 139.4(\mathrm{C}), 138.8(\mathrm{C}), 129.5\left(\mathrm{C}_{\mathrm{Ph}}\right), 129.4(\mathrm{CH}), 128.9(\mathrm{CH})$, $128.8(\mathrm{CH}), 126.0(\mathrm{CH}), 123.3(\mathrm{CH}), 123.1(\mathrm{CH}), 121.3(\mathrm{CH}), 115.6(\mathrm{CN})$, $76.1(\mathrm{~d}, J=5.1 \mathrm{~Hz}, \mathrm{OCH}), 59.3(\mathrm{C}), 38.5\left(\mathrm{CH}_{2}\right), 38.2\left(\mathrm{CH}_{2}\right), 30.6\left(\mathrm{CH}_{2}\right), 27.7$ $\left(\mathrm{d}, J=7.1 \mathrm{~Hz}, \mathrm{CH}_{2} \mathrm{CN}\right)$; IR : $v_{\max }=3013,2956,2855,1616,1584,1466,1294$, 1222, 1159, 1132, 1054, 1014, 998, 931, 906, 790, 755; HR-MS (ESI): $\mathrm{m} / \mathrm{z}$ calcd. for $\mathrm{C}_{26} \mathrm{H}_{22} \mathrm{NO}_{4} \mathrm{PNa}[\mathrm{M}+\mathrm{Na}]^{+}$, 466.1184. Found, 466.1176. $[\alpha] \mathrm{D}=$ $+164\left(c=1, \mathrm{CHCl}_{3}\right)$

Synthesis of phosphates 14a,a'. To a solution of rac-VANOL ( $66 \mathrm{mg}, 0.15$ $\mathrm{mmol})$ and $1 H$-tetrazole $(44 \mathrm{mg}, 0.63 \mathrm{mmol})$ in anhydrous DCM $(7.5 \mathrm{~mL})$ was added dropwise a solution of ethyl 3-((bis(diisopropylamino)phosphino)oxy)-4-cyanobutanoate $(R)-7$ (70 $\mathrm{mg}, 0.18 \mathrm{mmol}, 1.2 \mathrm{eq}$.) in anhydrous DCM $(2 \mathrm{~mL})$. The mixture was heated at $40{ }^{\circ} \mathrm{C}$ for $5 \mathrm{~h}$. The reaction was then quenched by the addition of saturated aqueous $\mathrm{NaHCO}_{3}$ $(20 \mathrm{~mL})$ and extracted twice with $\mathrm{CH}_{2} \mathrm{Cl}_{2}$. The organic phase was dried over $\mathrm{MgSO}_{4}$ and the solvents were removed under reduced pressure $\left({ }^{31} \mathrm{P}\right.$ NMR (202.5 MHz, $\left.\mathrm{CDCl}_{3}\right): \delta=143.6$ and $143.4 \mathrm{ppm}$ ). The mixture of phosphites was dissolved in $\mathrm{CH}_{2} \mathrm{Cl}_{2}(1 \mathrm{~mL})$. TBHP (5.5M in decane, $82 \mu \mathrm{L}, 0.45 \mathrm{mmol}$ ) was added to the solution at $0{ }^{\circ} \mathrm{C}$. Then the reaction mixture was allowed to warm to room temperature and stirred for 30 minutes. The reaction was treated with saturated aqueous $\mathrm{Na}_{2} \mathrm{~S}_{2} \mathrm{O}_{3}$. The layers were separated, the organic layer was dried over $\mathrm{MgSO}_{4}$ and concentrated in vacuo. The crude mixture was purified by chromatography on silica gel (heptane/EtOAc $=6: 4$ to $4: 6$ ) to afford $62 \mathrm{mg}$ ( $65 \%$ yield) of 1:1 mixture of diastereoisomers. The phosphates 14a and 14a' were separated by semi-preparative HPLC on a $\mathrm{SiO}_{2}$ column ( $\left.250 \times 10 \mathrm{~mm}, 5 \mathrm{mic}\right)$, heptane/EtOAc gradient (8:2 to 4:6). 10a: retention time $31.3 \mathrm{~min}$, (22 $\mathrm{mg}, 35 \%$ yield); 14a': retention time 31.6 min, (20 mg, $32 \%$ yield)

14a: ${ }^{31} \mathrm{P}$ NMR $\left(202.5 \mathrm{~Hz}, \mathrm{CDCl}_{3}\right): \delta=1.4 \mathrm{ppm} ;{ }^{1} \mathrm{H}$ NMR $(500.1 \mathrm{MHz}$, $\left.\mathrm{CDCl}_{3}\right): \delta 8.44(\mathrm{~d}, J=8.5 \mathrm{~Hz}, 1 \mathrm{H}), 8.30(\mathrm{~d}, J=8.5 \mathrm{~Hz}, 1 \mathrm{H}), 7.87(\mathrm{~d}, J=8.0$ $\mathrm{Hz}, 1 \mathrm{H}), 7.84(\mathrm{~d}, J=8.0 \mathrm{~Hz}, 1 \mathrm{H}), 7.70(\mathrm{t}, J=7.6 \mathrm{~Hz}, 1 \mathrm{H}), 7.66-7.60(\mathrm{~m}, 3 \mathrm{H})$, $7.53(\mathrm{~s}, 2 \mathrm{H}), 7.12(\mathrm{~m}, 2 \mathrm{H}), 6.96-6.91(\mathrm{~m}, 4 \mathrm{H}), 6.49-6.46(\mathrm{~m}, 4 \mathrm{H}), 5.49-5.43$ $(\mathrm{m}, 1 \mathrm{H}, \mathrm{OCH}), 4.24\left(\mathrm{q}, J=7.2 \mathrm{~Hz}, 2 \mathrm{H}, \mathrm{OCH}_{2}\right), 3.09(\mathrm{dd}, J=17.0,5.0 \mathrm{~Hz}$, $1 \mathrm{H}), 2.98-2.93(\mathrm{~m}, 2 \mathrm{H}), 2.85\left(\mathrm{dd}, J=17.0,7.5 \mathrm{~Hz}, 1 \mathrm{H}, \mathrm{CH}_{2}\right), 1.31(\mathrm{t}, J=7.2$ $\mathrm{Hz}, 3 \mathrm{H}) ;{ }^{13} \mathrm{C}$ NMR $\left(125.8 \mathrm{MHz}, \mathrm{CDCl}_{3}\right): \delta=168.7(\mathrm{CO}), 145.8(\mathrm{~d}, J=12.0$ Hz, O-C), 144.7 (d, J = 8.3 Hz, O-C), 140.4 (C), 140.1 (C), 139.8 (C), 134.5 $(\mathrm{C}), 134.3(\mathrm{C}), 129.2(\mathrm{CH}), 129.1(\mathrm{CH}), 128.2(\mathrm{CH}), 128.0(\mathrm{CH}), 127.9(\mathrm{CH})$, $127.9(\mathrm{CH}), 127.7(\mathrm{CH}), 127.6(\mathrm{CH}), 127.4(\mathrm{CH}), 127.2(\mathrm{CH}), 126.9(\mathrm{CH})$, $126.8(\mathrm{CH}), 125.7$ (C), 125.2 (d, $J=2.8 \mathrm{~Hz}, \mathrm{C}), 122.6(\mathrm{C}), 122.5(\mathrm{CH}), 121.9$ $(\mathrm{CH}), 115.3(\mathrm{CN}), 72.2(\mathrm{~d}, J=4.7 \mathrm{~Hz}, \mathrm{OCH}), 61.7\left(\mathrm{CH}_{2}\right), 39.1(\mathrm{~d}, J=6.4$ $\left.\mathrm{Hz}, \mathrm{CH}_{2}\right), 24.5\left(\mathrm{CH}_{2}\right), 14.3\left(\mathrm{CH}_{3}\right)$; IR: $v_{\max }=3054,3028,2986,1737,1489$, $1362,1301,1290,1025,762,700$; HR-MS (ESI) : $\mathrm{m} / z$ calcd. for $\mathrm{C}_{39} \mathrm{H}_{31} \mathrm{NO}_{6} \mathrm{P}$ $[\mathrm{M}+\mathrm{H}]^{+}, 640.1889$. Found: $640.1880 ;[\alpha]_{\mathrm{D}}^{20}=+51\left(\mathrm{c}=1, \mathrm{CHCl}_{3}\right)$.

14a': ${ }^{31} \mathrm{P}$ NMR $\left(202.5 \mathrm{~Hz}, \mathrm{CDCl}_{3}\right): \delta=1.7 \mathrm{ppm} ;{ }^{1} \mathrm{H} \mathrm{NMR}(500.1 \mathrm{MHz}$, $\left.\mathrm{CDCl}_{3}\right): \delta=8.45(\mathrm{~d}, J=8.5 \mathrm{~Hz}, 1 \mathrm{H}), 8.38(\mathrm{~d}, J=8.5 \mathrm{~Hz}, 1 \mathrm{H}), 7.85(\mathrm{~m}, 2 \mathrm{H})$, $7.85(\mathrm{~m}, 2 \mathrm{H})$, ), $7.71(\mathrm{t}, J=7.6 \mathrm{~Hz}, 2 \mathrm{H}), 7.65-7.61(\mathrm{~m}, 2 \mathrm{H}), 7.53(\mathrm{~s}, 1 \mathrm{H}), 7.52$ (s, 1H), 7.13-7.09 (m, 2H), 6.95-6.92 (m, 4H), 6.47-6.46 (m, 4H), 5.52-5.45 (m, $1 \mathrm{H}, \mathrm{OCH}), 4.12\left(\mathrm{q}, J=7.5 \mathrm{~Hz}, 2 \mathrm{H}, \mathrm{OCH}_{2}\right), 3.13(\mathrm{dd}, J=17.0,5.4 \mathrm{~Hz}$, $1 \mathrm{H}), 3.02-2.97(\mathrm{~m}, 2 \mathrm{H}), 2.86(\mathrm{dd}, J=17.0,7.0 \mathrm{~Hz}, 1 \mathrm{H}), 1.19(\mathrm{t}, J=7.5 \mathrm{~Hz}$, $3 \mathrm{H}) ;{ }^{13} \mathrm{C}$ NMR $\left(125.8 \mathrm{MHz}, \mathrm{CDCl}_{3}\right): \delta=168.7(\mathrm{CO}), 145.9(\mathrm{~d}, J=12.0 \mathrm{~Hz}$, O-C), 144.7 (d, $J=9.2 \mathrm{~Hz}, \mathrm{O}-\mathrm{C}), 140.2$ (C), 140.1 (C), 139.9 (C), 139.8 (C), 134.5 (C), $134.3(\mathrm{C}), 129.1(\mathrm{CH}), 129.1(\mathrm{CH}), 128.1(\mathrm{CH}), 128.0(\mathrm{CH}), 127.9$ $(\mathrm{CH}), 127.7(\mathrm{CH}), 127.6(\mathrm{CH}), 127.5(\mathrm{CH}), 127.4(\mathrm{CH}), 126.8(\mathrm{C}), 126.8(\mathrm{C})$, $125.8(\mathrm{~d}, J=1.8 \mathrm{~Hz}, \mathrm{C}), 125.3(\mathrm{~d}, J=2.8 \mathrm{~Hz}, \mathrm{C}), 122.7(\mathrm{C}), 122.5(\mathrm{CH}), 122.3$ (C), $122.1(\mathrm{CH}), 115.3(\mathrm{CN}), 72.2(\mathrm{~d}, J=4.7 \mathrm{~Hz}, \mathrm{OCH}), 61.5\left(\mathrm{CH}_{2}\right), 39.0(\mathrm{~d}$, $\left.J=4.5 \mathrm{~Hz}, \mathrm{CH}_{2}\right), 24.7\left(\mathrm{~d}, J=5.5 \mathrm{~Hz}, \mathrm{CH}_{2}\right), 14.2\left(\mathrm{CH}_{3}\right) ; \mathrm{IR}: v_{\max }=3055$, 3026, 2985, 2929, 1735, 1489, 1363, 1301, 1290, 1024, 967, 946, 762, 700; HR-MS (ESI) $)_{20}: m / z$ calcd. for $\mathrm{C}_{39} \mathrm{H}_{31} \mathrm{NO}_{6} \mathrm{P}[\mathrm{M}+\mathrm{H}]^{+}, 640.1889$. Found, $640.1882 ;[\alpha]_{\mathrm{D}}^{20}=-89\left(c=1, \mathrm{CHCl}_{3}\right)$

Phosphates 15a,a'. Phosphates 15 have been prepared from rac-VANOL and diethyl (S)-malate $N, N, N^{\prime}, N^{\prime}$-tetraisopropylphosphorodiamidite (S)-9 as described for 14a,a'. The corresponding phosphites were obtained after $16 \mathrm{~h}$ heating at $40^{\circ} \mathrm{C}\left({ }^{31} \mathrm{P} \mathrm{NMR}\left(202.5 \mathrm{MHz}, \mathrm{CDCl}_{3}\right): \delta=148.4\right.$ and $\left.144.3 \mathrm{ppm}\right)$. After oxidation, the crude mixture was purified by chromatography on silica gel (heptane/EtOAc $=6: 4$ to $4: 6,72 \%$ yield). Phosphates 15a and 15a' were separated by semi-preparative HPLC on a Sunfire C18 reversed phase column, $\mathrm{H}_{2} \mathrm{O} / \mathrm{MeCN} /$ Formic Acid (23/77/0.1). 15a: retention time $16.6 \mathrm{~min}$; 15a': retention time $17.8 \mathrm{~min}$.

15a: ${ }^{31} \mathrm{P}$ NMR $\left(202.5 \mathrm{~Hz}, \mathrm{CDCl}_{3}\right): \delta=2.0 \mathrm{ppm} ;{ }^{1} \mathrm{H}$ NMR $(500.1 \mathrm{MHz}$, $\left.\mathrm{CDCl}_{3}\right): \delta=8.65(\mathrm{~d}, J=8.4 \mathrm{~Hz}, 1 \mathrm{H}), 8.51(\mathrm{~d}, J=8.4 \mathrm{~Hz}, 1 \mathrm{H}), 7.83(\mathrm{~d}, J=$ $8.1 \mathrm{~Hz}, 2 \mathrm{H}), 7.71-7.65(\mathrm{~m}, 2 \mathrm{H}), 7.62-7.59(\mathrm{~m}, 2 \mathrm{H}), 7.51(\mathrm{~s}, 1 \mathrm{H}), 7.50(\mathrm{~s}, 1 \mathrm{H})$, 7.12-7.08 (m, 2H), $6.93(\mathrm{~m}, 4 \mathrm{H}), 6.48-6.46(\mathrm{~m}, 4 \mathrm{H}), 5.71-5.67(\mathrm{~m}, 1 \mathrm{H}, \mathrm{OCH})$, 4.37 (q, $J=7.2 \mathrm{~Hz}, 2 \mathrm{H}, \mathrm{OCH}_{2}$ ), 4.11 (q, $\left.J=7.2 \mathrm{~Hz}, 2 \mathrm{H}, \mathrm{OCH}_{2}\right), 3.05-3.00$ (m, 1H), 2.95-2.90 (m, 1H), 1.38 (t, $J=7.2 \mathrm{~Hz}, 3 \mathrm{H}, \mathrm{Me}), 1.15$ (t, $J=7.2 \mathrm{~Hz}$, $3 \mathrm{H}, \mathrm{Me}) ;{ }^{13} \mathrm{C}$ NMR $\left(125.8 \mathrm{MHz}, \mathrm{CDCl}_{3}\right): \delta=168.8(\mathrm{CO}), 168.4$ (d, $J=3.6$ $\mathrm{Hz}, \mathrm{CO}$ ), 146.3 (d, $J=11.8 \mathrm{~Hz}, \mathrm{O}-\mathrm{C}$ ), 144.9 (d, $J=9.2 \mathrm{~Hz}, \mathrm{O}-\mathrm{C}$ ), 140.2 (C), $140.1(\mathrm{C}), 140.0(\mathrm{C}), 139.9$ (C), $134.5(\mathrm{C}), 134.3(\mathrm{C}), 129.2(\mathrm{CH}), 129.1(\mathrm{CH})$, $127.9(\mathrm{CH}), 127.8(\mathrm{CH}), 127.7(\mathrm{CH}), 127.6(\mathrm{CH}), 127.5(\mathrm{CH}), 127.2(\mathrm{CH})$, $126.9(\mathrm{CH}), 126.7(\mathrm{CH}), 126.0(\mathrm{C}), 125.6(\mathrm{~d}, J=2.8 \mathrm{~Hz}, \mathrm{C}), 123.2(\mathrm{CH})$, $122.8(\mathrm{CH}), 122.3(\mathrm{C}), 74.3(\mathrm{~d}, J=5.3 \mathrm{~Hz}, \mathrm{OCH}), 62.6\left(\mathrm{OCH}_{2}\right), 61.4\left(\mathrm{OCH}_{2}\right)$, $37.9\left(\mathrm{~d}, J=5.4 \mathrm{~Hz}, \mathrm{CH}_{2}\right), 14.3\left(\mathrm{CH}_{3}\right), 14.2\left(\mathrm{CH}_{3}\right) ; \mathrm{IR}: v_{\max }=3056,2985$, 2937, 1760, 1738, 1489, 1363, 1305, 1291, 1181, 1110, 1048, 1029, 761, 700; HR-MS (ESI): $\mathrm{m} / z$ calcd. for $\mathrm{C}_{40} \mathrm{H}_{34} \mathrm{O}_{8} \mathrm{P}[\mathrm{M}+\mathrm{H}]^{+}, 673.1991$. Found, $673.2017 ;[\alpha]_{\mathrm{D}}^{20}=-43\left(c=1, \mathrm{CHCl}_{3}\right)$.

15a': ${ }^{31} \mathrm{P}$ NMR $\left(202.5 \mathrm{~Hz}, \mathrm{CDCl}_{3}\right): \delta=2.3 \mathrm{ppm} ;{ }^{1} \mathrm{H}$ NMR $(500.1 \mathrm{MHz}$, $\left.\mathrm{CDCl}_{3}\right): \delta=8.51(\mathrm{~d}, J=8.2 \mathrm{~Hz}, 1 \mathrm{H}), 8.32(\mathrm{~d}, J=8.2 \mathrm{~Hz}, 1 \mathrm{H}), 7.84-7.82(\mathrm{~m}$, $2 \mathrm{H}), 7.68(\mathrm{t}, J=7.8 \mathrm{~Hz}, 1 \mathrm{H}), 7.64-7.57(\mathrm{~m}, 3 \mathrm{H}), 7.52(\mathrm{~s}, 1 \mathrm{H}), 7.49(\mathrm{~s}, 1 \mathrm{H})$, 7.12-7.08 (m, 2H), 6.95-6.91 (m, 4H), 6.49-6.46 (m, 4H), 5.76-5.71 (m, $1 \mathrm{H}$, $\mathrm{OCH}), 4.41-4.33\left(\mathrm{~m}, 2 \mathrm{H}, \mathrm{OCH}_{2}\right), 4.19-4.11\left(\mathrm{~m}, 2 \mathrm{H}, \mathrm{O} \mathrm{CH}_{2}\right), 3.05$ (dd, $J=$ $\left.16.5,4.8 \mathrm{~Hz}, 1 \mathrm{H}, \mathrm{CH}_{2}\right), 2.96\left(\mathrm{dd}, J=16.5,6.4 \mathrm{~Hz}, 1 \mathrm{H}, \mathrm{CH}_{2}\right), 1.36(\mathrm{t}, J=7.1$ $\mathrm{Hz}, 3 \mathrm{H}, \mathrm{Me}), 1.19$ (t, $J=7.1 \mathrm{~Hz}, 3 \mathrm{H}, \mathrm{Me}) ;{ }^{13} \mathrm{C} \mathrm{NMR}\left(125.8 \mathrm{MHz}, \mathrm{CDCl}_{3}\right)$ : $\delta=168.7(\mathrm{CO}), 146.0(\mathrm{~d}, J=11.9 \mathrm{~Hz}, \mathrm{O}-\mathrm{C}), 145.0(\mathrm{~d}, J=8.2 \mathrm{~Hz}, \mathrm{O}-\mathrm{C})$, 140.3 (C), 140.1 (C), 140.0 (C), 139.9 (C), 134.5 (C), 134.3 (C), $129.2(\mathrm{CH})$, $129.1(\mathrm{CH}), 128.0(\mathrm{CH}), 127.9(\mathrm{CH}), 127.9(\mathrm{CH}), 127.6(\mathrm{CH}), 127.5(\mathrm{CH})$ $127.5(\mathrm{CH}), 127.2(\mathrm{CH}), 127.0(\mathrm{CH}), 126.8(\mathrm{CH}) 126.7(\mathrm{CH}), 126.1(\mathrm{~d}, J=$ $1.8 \mathrm{~Hz}, \mathrm{C}), 125.5$ (d, $J=2.6 \mathrm{~Hz}, \mathrm{C}), 122.8$ (CH), 122.7 (C), 122.5 (C), 122.3 $(\mathrm{CH}), 74.2(\mathrm{~d}, J=5.4 \mathrm{~Hz}, \mathrm{OCH}), 62.7\left(\mathrm{OCH}_{2}\right), 61.4\left(\mathrm{OCH}_{2}\right), 37.9(\mathrm{~d}, J=7.4$ $\left.\mathrm{Hz}, \mathrm{CH}_{2}\right), 14.3\left(\mathrm{CH}_{3}\right), 14.2\left(\mathrm{CH}_{3}\right) ; \mathrm{IR}: v_{\max }=2986,2930,1740,1489,1364$, 1306, 1291, 1262, 1182, 1110, 1049, 1030, 762, 751, 700; HR-MS (ESI) $\mathrm{m} / \mathrm{z}$ calcd. for $\mathrm{C}_{40} \mathrm{H}_{34} \mathrm{O}_{8} \mathrm{P}[\mathrm{M}+\mathrm{H}]^{+}, 673.1991$. Found, 673.2001. $[\alpha]_{\mathrm{D}}^{20}=+95$ $\left(c=1, \mathrm{CHCl}_{3}\right)$.

Phosphates 16a,a'. Phosphates 16 have been prepared from rac-VANOL $(0.2 \mathrm{mmol})$ and $N$-(tert-Butoxycarbonyl)-L-threonine methyl ester $N, N, N$, $N$ '-tetraisopropylphosphorodiamidite $(\mathbf{2} \boldsymbol{S}, \mathbf{3 R}) \mathbf{- 1 0}$, as described for 14a,a'. The corresponding phosphites $\left({ }^{31} \mathrm{P}\right.$ NMR: $\delta=154.3$ and $142.3 \mathrm{ppm}$ ppm) were obtained after $16 \mathrm{~h}$ heating at $40^{\circ} \mathrm{C}$. After oxidation, the crude mixture was purified by chromatography on silica gel (heptane/EtOAc $=6: 4$ 
to $4: 6,78 \%$ yield). Phosphates $\mathbf{1 6 a}$ and $\mathbf{1 6 a}$ ' were separated by semipreparative HPLC on a Sunfire $\mathrm{C} 18$ column, $\mathrm{H}_{2} \mathrm{O} / \mathrm{MeCN} /$ formic acid (20/80/0.1). 16a: retention time $12.0 \mathrm{~min}$; 16a': retention time $15.0 \mathrm{~min}$ 16a: ${ }^{31} \mathrm{P}$ NMR $\left(202.5 \mathrm{~Hz}, \mathrm{CDCl}_{3}\right): \delta=1.6 \mathrm{ppm} ;{ }^{1} \mathrm{H}$ NMR $(500.1 \mathrm{MHz}$, $\left.\mathrm{CDCl}_{3}\right): \delta 8.44(\mathrm{~d}, J=8.1 \mathrm{~Hz}, 1 \mathrm{H}), 8.32(\mathrm{~d}, J=7.8 \mathrm{~Hz}, 1 \mathrm{H}), 7.88(\mathrm{~d}, J=8.0$ $\mathrm{Hz}, 1 \mathrm{H}), 7.83(\mathrm{~d}, J=8.0 \mathrm{~Hz}, 1 \mathrm{H}), 7.71-7.59(\mathrm{~m}, 4 \mathrm{H}), 7.53(\mathrm{~s}, 1 \mathrm{H}), 7.51(\mathrm{~s}$, $1 \mathrm{H}), 7.13-7.08(\mathrm{~m}, 2 \mathrm{H}), 6.95-6.91(\mathrm{~m}, 4 \mathrm{H}), 6.47$ (d, $J=7.6 \mathrm{~Hz}, 4 \mathrm{H}), 5.54-$ $5.49(\mathrm{~m}, 1 \mathrm{H}, \mathrm{OCH}), 4.93(\mathrm{~d}, J=9.6 \mathrm{~Hz}, 1 \mathrm{H}), 4.44(\mathrm{~d}, J=9.6 \mathrm{~Hz}, 1 \mathrm{H}), 3.56$ (s, 3H, OMe), $1.65(\mathrm{~d}, J=6.3 \mathrm{~Hz}, 3 \mathrm{H}), 1.42\left(\mathrm{~s}, 9 \mathrm{H}, \mathrm{CMe}_{3}\right) ;{ }^{13} \mathrm{C}$ NMR $(125.8$ $\mathrm{MHz}, \mathrm{CDCl}_{3}$ ) : $\delta=169.9(\mathrm{CO}), 155.9(\mathrm{CO}), 146.0$ (d, $\left.J=11.1 \mathrm{~Hz}, \mathrm{O}-\mathrm{C}\right)$, 145.0 (d, $J=8.3 \mathrm{~Hz}, \mathrm{O}-\mathrm{C}), 140.3$ (C), 140.1 (C), 139.9 (C), 139.8 (C), 134.5 (C), 134.4 (C), $129.1(\mathrm{CH}), 128.1(\mathrm{CH}), 127.9(\mathrm{CH}), 127.8(\mathrm{CH}), 127.5(\mathrm{CH})$, $127.4(\mathrm{CH}), 127.2(\mathrm{CH}), 127.0(\mathrm{CH}), 126.8(\mathrm{CH}), 126.7(\mathrm{CH}), 125.9(\mathrm{C})$, $125.4(\mathrm{C}), 122.7(\mathrm{C}), 122.6(\mathrm{CH}), 122.5(\mathrm{C}), 122.2(\mathrm{C}), 80.4\left(\mathrm{CMe}_{3}\right), 78.4$ (d, $J=5.4 \mathrm{~Hz}, \mathrm{O}-\mathrm{CH}), 58.0(\mathrm{~d}, J=5.5 \mathrm{~Hz}, \mathrm{CH}), 52.7\left(\mathrm{OCH}_{3}\right), 28.4\left(\mathrm{CMe}_{3}\right), 19.1$ $\left(\mathrm{CH}_{3}\right)$; IR : $v_{\max }=3426,3057,3009,2980,1749,1718,1490,1364,1301$, 1290, 1165, 1017, 968, 761, 700; HR-MS (ESI) : $m / z$ calcd. for $\mathrm{C}_{42} \mathrm{H}_{39} \mathrm{NO}_{8} \mathrm{P}$ $[\mathrm{M}+\mathrm{H}]^{+}, 716.2413$. Found, 716.2431; $[\alpha] \mathrm{D}=-77\left(c=1, \mathrm{CHCl}_{3}\right)$.

16a': ${ }^{31} \mathrm{P}$ NMR $\left(202.5 \mathrm{~Hz}, \mathrm{CDCl}_{3}\right): \delta=2.1 \mathrm{ppm} ;{ }^{1} \mathrm{H}$ NMR $(500.1 \mathrm{MHz}$, $\left.\mathrm{CDCl}_{3}\right): \delta=8.47(\mathrm{~d}, J=8.4 \mathrm{~Hz}, 1 \mathrm{H}), 8.36(\mathrm{~d}, J=8.4 \mathrm{~Hz}, 1 \mathrm{H}), 7.87(\mathrm{~d}, J=$ $8.0 \mathrm{~Hz}, 1 \mathrm{H}), 7.84(\mathrm{~d}, J=8.5 \mathrm{~Hz}, 1 \mathrm{H}), 7.79(\mathrm{t}, J=7.5 \mathrm{~Hz}, 1 \mathrm{H}), 7.69-7.59(\mathrm{~m}$, $3 \mathrm{H}), 7.53(\mathrm{~s}, 1 \mathrm{H}), 7.52(\mathrm{~s}, 1 \mathrm{H}), 7.13-7.09(\mathrm{~m}, 2 \mathrm{H}), 6.95-6.92(\mathrm{~m}, 4 \mathrm{H}), 6.49-$ $6.46(\mathrm{~m}, 4 \mathrm{H}), 5.60-5.54(\mathrm{~m}, 1 \mathrm{H}, \mathrm{OCH}), 5.06(\mathrm{~d}, J=9.6 \mathrm{~Hz}, 1 \mathrm{H}), 4.49(\mathrm{~d}, J=$ $9.6 \mathrm{~Hz}, 1 \mathrm{H}), 3.91$ (s, $3 \mathrm{H}, \mathrm{OMe}), 1.46(\mathrm{~d}, J=6.6 \mathrm{~Hz}, 3 \mathrm{H}, \mathrm{Me}), 1.42(\mathrm{~s}, 9 \mathrm{H}$, $\left.\mathrm{CMe}_{3}\right) ;{ }^{13} \mathrm{C}$ NMR $\left(125.8 \mathrm{MHz}, \mathrm{CDCl}_{3}\right): \delta=170.5(\mathrm{CO}), 156.0(\mathrm{CO}), 146.1$ (d, $J=12.0 \mathrm{~Hz}, \mathrm{O}-\mathrm{C}$ ), 145.2 (d, $J=9.2 \mathrm{~Hz}, \mathrm{O}-\mathrm{C}), 140.3$ (C), 140.1 (C), 139.9 (C), $139.8(\mathrm{C}), 134.5(\mathrm{C}), 134.3(\mathrm{C}), 129.1(\mathrm{CH}), 128.1(\mathrm{CH}), 128.0(\mathrm{CH})$, $127.9(\mathrm{CH}), 127.6(\mathrm{CH}), 127.5(\mathrm{CH}), 127.3(\mathrm{CH}), 126.8(\mathrm{CH}), 126.7(\mathrm{CH})$, 125.7 (C), $125.5(\mathrm{C}), 122.7(\mathrm{C}), 122.5(\mathrm{CH}), 122.4(\mathrm{CH}), 80.4\left(\mathrm{CMe}_{3}\right), 78.6$ $(\mathrm{d}, J=5.5 \mathrm{~Hz}, \mathrm{OCH}), 58.1(\mathrm{~d}, J=5.5 \mathrm{~Hz}, \mathrm{CH}), 53.1\left(\mathrm{OCH}_{3}\right), 28.4\left(\mathrm{CMe}_{3}\right)$, $18.9\left(\mathrm{CH}_{3}\right)$; IR : $v_{\max }=3430,3056,2979,1752,1721,1490,1363,1302,1291$, 1165, 1017, 968, 761, 700; HR-MS (ESI): $\mathrm{m} / \mathrm{z}$ calcd. for $\mathrm{C}_{42} \mathrm{H}_{39} \mathrm{NO}_{8} \mathrm{P}$ $[\mathrm{M}+\mathrm{H}]^{+}, 716.2413$. Found, 716.2441; $[\alpha]_{\mathrm{D}}=+118,5\left(c=1, \mathrm{CHCl}_{3}\right)$.

Representative procedure for the removal of the chiral auxiliary. Phosphate 16a (36 mg, $0.05 \mathrm{mmol}$ ) was dissolved in DCM (1 mL) and DBU $(15 \mu \mathrm{L}, 0.10 \mathrm{mmol})$ was added at room temperature. After $20 \mathrm{~min}$, the crude 7b. DBU salt was purified by column chromatography (eluent: DCM/MeOH gradient, from 100:0 to 90:10). The fractions were collected, concentrated in vacuo and diluted in DCM $(10 \mathrm{~mL})$. This solution was treated with $\mathrm{HCl} 6 \mathrm{~N}$ $\left(3^{*} 10 \mathrm{~mL}\right)$. The layers were separated and the organic layer was dried and concentrated in vacuo to afford the chiral phosphoric acid $(\boldsymbol{R})-7 \mathbf{b}(25 \mathrm{mg}$, 98\% yield). Ee $98.8 \%$ by HPLC: CHIRALPAK® ID column, THF $n-$ heptane 70/30, 0.3\% TEA, 0.5\% TFA, $4.7 \mathrm{~mL} / \mathrm{min}$. Retention times $5.7 \mathrm{~min}$, for $(R)-7 \mathbf{b}$ and $8.0 \mathrm{~min}$, for $(S)-7 \mathbf{b}$

Supporting Information. ${ }^{1} \mathrm{H},{ }^{31} \mathrm{P}$ and ${ }^{13} \mathrm{C}$ NMR spectra for the new compounds; X-ray data for $\mathbf{6 d}$.

\section{Acknowledgments}

This work has been carried out with the financial support of the Agence Nationale de la Recherche, within the ANR Blanc 'Chiracid' project, and the COST action 'ORganoCAtalysis' CM0905.

[1] a) J. Nielsen, O. Dahl, Nucleic Acids Res. 1987, 15, 3626; b) S. L. Beaucage, e-Eros 2003, DOI: 10.1002/047084289X.m047000312.

[2] For recent examples of synthetic uses of 1, see: a) C. E. Martin, F. Broecker, S. Eller, M. A. Oberli, C. Anish, C. L. Pereira, P. H. Seeberger, Chem. Commun. 2013, 49, 7159-7161; b) A. M. Varizhuk, D. N. Kaluzhny, R. A. Novikov, A. O. Chizhov, I. P. Smirnov, A. N. Chuvilin, O. N. Tatarinova, G. Y. Fisunov, G. E. Pozmogova, V. L. Florentiev, J. Org. Chem. 2013, 78, 5964-5969; c) Y. Ochi, O.
Nakagawa, K. Sakaguchi, S. Wada, H. Urata, Chem. Commun. 2013 49, 7620-7622; d) M. S. Noé, R. W. Sinkeldam, Y. Tor, J. Org. Chem 2013, 78, 8123-8128; e) G. Mathis, S. Bourg, S. Aci-Sèche, J.-C. Truffert, U. Asseline, Org. Biomol. Chem. 2013, 11, 1345-1357; f) M. T. Migawa, T. P. Prakash, G. Vasquez, P. P. Seth, E. E. Swayze, Org Lett. 2013, 15, 4316-4319; g) M. M. Piperakis, J. W. Gaynor, J. Fisher, R. Cosstick, Org. Biomol. Chem. 2013, 11, 966-974; h) K. Yamada, Y. Hattori, T. Inde, T. Kanamori, A. Ohkubo, K. Seio, M. Sekine, Bioorg. Med. Chem. Lett. 2013, 23, 776-778; i) Y. Hari, T. Osawa, Y. Kotobuki, A. Yahara, A. R. Shrestha, S. Obika, Bioorg. Med. Chem. 2013, 21, 4405-4412; j) C. M. Gampe, H. Tsukamoto, E. H. Doud, S. Walker, D. Kahne, J. Am. Chem. Soc. 2013, 135, 3776-3779.

[3] a) J. Stemper, K. Isaac, V. Duret, P. Retailleau, A. Voituriez, J.-F. Betzer, A. Marinetti, Chem. Commun. 2013, 49, 6084-6086; b) J. Stemper, K. Isaac, J. Pastor, P. Retailleau, A. Voituriez, J.-F. Betzer, A. Marinetti, Adv. Synth. Catal. 2013, 355, 3613-3624.

[4] T. Akiyama, J. Itoh, K. Fuchibe, Adv. Synth. Catal. 2006, 348, 9991010 .

[5] a) T. Akiyama, Chem. Rev. 2007, 107, 5744-5758; b) M. Terada Chem. Commun. 2008, 4097-4112; c) M. Terada, Bull. Chem. Soc. Jpn. 2010, 83, 101-119; d) A. Zamfir, S. Schenker, M. Freund, S. B. Tsogoeva, Org. Biomol. Chem. 2010, 8, 5262-5276; e) J. Yu, F. Shi, L.-Z. Gong, Acc. Chem. Res. 2011, 44, 1156-1171; f) S. Schenker, A. Zamfir, M. Freund, S. B. Tsogoeva, Eur. J. Org. Chem. 2011, 22092222; g) M. Rueping, B. J. Nachtsheim, W. Ieawsuwan, I. Atodiresei, Angew. Chem. Int. Ed. 2011, 50, 6706-6720; h) R. J. Phipps, G. L. Hamilton, F. D. Toste, Nat. Chem. 2012, 4, 603; i) K. Brak, E. N. Jacobsen, Angew. Chem. Int. Ed. 2013, 52, 534-561; j) M. Mahlau, B. List, Angew. Chem. Int. Ed. 2013, 52, 518-533.

[6] a) V. B. Birman, A. L. Rheingold, K.-C. Lam, Tetrahedron Asymmetry 1999, 10, 125-131; b) F. Xu, D. Huang, C. Han, W. Shen, X. Lin, Y. Wang, J. Org. Chem. 2010, 75, 8677-8680.

[7] a) B.-Q. Gong, W.-Y. Chen, B.-F. Hu, J. Org. Chem. 1991, 56, 423 425; b) P. Wipf, J.-K. Jung, J. Org. Chem. 2000, 65, 6319-6337; c) M. Yamanaka, J. Itoh, K. Fuchibe, T. Akiyama, J. Am. Chem. Soc. 2007, 129, 6756-6764.

[8] a) A. A. Desai, W. D. Wulff, Synthesis 2010, 3670-3680; b) Y. Zhang, S.-M. Yeung, H. Wu, D. P. Heller, C. Wu, W. D. Wulff, Org. Lett. 2003, 5, 1813-1816; c) G. Hu, D. Holmes, B. F. Gendhar, W. D. Wulff, J. Am. Chem. Soc. 2009, 131, 14355-14364.

[9] aJ. Bao, W. D. Wulff, J. B. Dominy, M. J. Fumo, E. B. Grant, A. C Rob, M. C. Whitcomb, S.-M. Yeung, R. L. Ostrander, A. L. Rheingold, J. Am. Chem. Soc. 1996, 118, 3392-3405; bZ. Ding, W. E. G. Osminski, H. Ren, W. D. Wulff, Org. Process Res. Dev. 2011, 15, 1089-1107.

[10] a) A. A. Desai, L. Huang, W. D. Wulff, G. B. Rowland, J. C. Antilla, Synthesis 2010, 2106-2109; b) G. B. Rowland, H. Zhang, E. B. Rowland, S. Chennamadhavuni, Y. Wang, J. C. Antilla, J. Am. Chem. Soc. 2005, 127, 15696-15697.

[11] a) H. Takaku, T. Watanabe, S. Hamamoto, Tetrahedron Lett. 1988, 29 , 81-84; b) P. G. Reddy, B.-K. Chun, H.-R. Zhang, S. Rachaconda, B S. Ross, M. J. Sofia, J. Org. Chem. 2011, 76, 3782-3790.

[12] NMR spectra for the epimeric mixtures of phosphates 11-13 are given as supporting information 
Various chiral phosphorodiamidites displaying $\beta$-cyanoethyl or $\beta$-(alkoxycarbonyl)ethyl chains behave as synthetic equivalents of the well-known $\beta$-cyanoethyl tetraisopropylphosphorodiamidite. They can be used notably for the synthesis and resolution of chiral phosphoric acids via chromatographic separations of diastereomeric mixtures.

\section{((Key Topic))}

Kévin Isaac, Jérémy Stemper, Pascal Retailleau, Jean-François Betzer,* Angela Marinetti*

Chiral Synthetic Equivalents of 2Cyanoethyl Tetraisopropylphosphorodiamidite: Application to the Synthesis and Resolution of Chiral Phosphoric Acids

Keywords: Chiral phosphorodiamidites / resolution / phosphoric acids / phosphates 